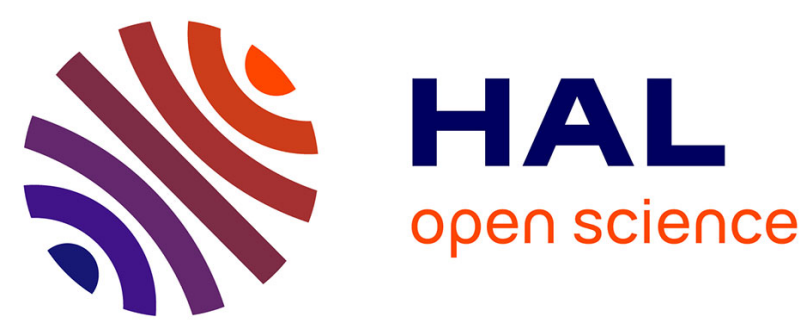

\title{
RhoG GTPase Controls a Pathway That Independently Activates Rac1 and Cdc42Hs
}

Cécile Gauthier-Rouvière, Emmanuel Vignal, Mayya Mériane, Pierre Roux, Philippe Montcourier, Philippe Fort

\section{To cite this version:}

Cécile Gauthier-Rouvière, Emmanuel Vignal, Mayya Mériane, Pierre Roux, Philippe Montcourier, et al.. RhoG GTPase Controls a Pathway That Independently Activates Rac1 and Cdc42Hs. Molecular Biology of the Cell, 1998, 9 (6), pp.1379-1394. 10.1091/mbc.9.6.1379 . hal-02267502

\section{HAL Id: hal-02267502 \\ https://hal.science/hal-02267502}

Submitted on 30 Jun 2020

HAL is a multi-disciplinary open access archive for the deposit and dissemination of scientific research documents, whether they are published or not. The documents may come from teaching and research institutions in France or abroad, or from public or private research centers.
L'archive ouverte pluridisciplinaire HAL, est destinée au dépôt et à la diffusion de documents scientifiques de niveau recherche, publiés ou non, émanant des établissements d'enseignement et de recherche français ou étrangers, des laboratoires publics ou privés.

\section{(1)(1) $\$(0)$}

Distributed under a Creative Commons Attribution - NonCommercial - ShareAlikel 4.0 


\title{
RhoG GTPase Controls a Pathway That Independently Activates Rac1 and Cdc42Hs
}

\author{
Cécile Gauthier-Rouvière, ${ }^{*+}$ Emmanuel Vignal, Mayya Mériane, ${ }^{\dagger}$ \\ Pierre Roux, Philippe Montcourier, ${ }^{\ddagger}$ and Philippe Fort
}

IGMM, CNRS-UMR5535, Route de Mende, 34293 Montpellier Cedex 05 France

Submitted January 14, 1998; Accepted March 27, 1998

Monitoring Editor: David Drubin

\begin{abstract}
RhoG is a member of the Rho family of GTPases that shares $72 \%$ and $62 \%$ sequence identity with Rac1 and Cdc42Hs, respectively. We have expressed mutant RhoG proteins fused to the green fluorescent protein and analyzed subsequent changes in cell surface morphology and modifications of cytoskeletal structures. In rat and mouse fibroblasts, green fluorescent protein chimera and endogenous RhoG proteins colocalize according to a tubular cytoplasmic pattern, with perinuclear accumulation and local concentration at the plasma membrane. Constitutively active RhoG proteins produce morphological and cytoskeletal changes similar to those elicited by a simultaneous activation of Rac1 and Cdc42Hs, i.e., the formation of ruffles, lamellipodia, filopodia, and partial loss of stress fibers. In addition, RhoG and Cdc42Hs promote the formation of microvilli at the cell apical membrane. RhoG-dependent events are not mediated through a direct interaction with Rac1 and Cdc42Hs targets such as PAK-1, POR1, or WASP proteins but require endogenous Rac1 and Cdc42Hs activities: coexpression of a dominant negative Rac1 impairs membrane ruffling and lamellipodia but not filopodia or microvilli formation. Conversely, coexpression of a dominant negative Cdc42Hs only blocks microvilli and filopodia, but not membrane ruffling and lamellipodia. Microtubule depolymerization upon nocodazole treatment leads to a loss of RhoG protein from the cell periphery associated with a reversal of the RhoG phenotype, whereas PDGF or bradykinin stimulation of nocodazole-treated cells could still promote Rac1- and Cdc42Hs-dependent cytoskeletal reorganization. Therefore, our data demonstrate that RhoG controls a pathway that requires the microtubule network and activates Rac1 and Cdc42Hs independently of their growth factor signaling pathways.
\end{abstract}

\section{INTRODUCTION}

The Rho family of Ras-like GTPases includes Rac $(1,2$, and 3), RhoG, Cdc42Hs, TC10, TTF/RhoH, Rho (A, B, and C), RhoD, RhoE, and RhoL. Like other Ras-related proteins, most of the Rho GTPases adopt either active GTP-bound or inactive GDP-bound conformational states. Specific substitutions based on Ras studies result in the expression of proteins which are either in a

\footnotetext{
* Corresponding author. E-mail address: gauthier@igm.cnrsmop.fr.

+ Present address: CRBM, CNRS-UPR1086, Route de Mende, 34293 Montpellier Cedex 05 France.

‡ Present address: CNRS-UMR 5539, Université de Montpellier II, 34095 Montpellier Cedex 05 France.
}

constitutively active GTP-bound (e.g., G12V) or dominant negative GDP-bound (e.g., T17N) conformations. By using such mutant proteins, Rho members have been implicated in many physiological processes such as the control of cell shape (Tapon and Hall, 1997), cell motility (Aepfelbacher et al., 1994, 1996), cell polarity (Adams et al., 1990), smooth muscle contraction (Hirata et al., 1992), cell adhesion (Nobes and Hall, 1995; Braga et al., 1997), and cell division (Dutartre et al., 1996). All of these events involve morphological changes associated with actin polymerization. In fibroblasts, lysophosphatidic acid-stimulated stress fiber formation requires RhoA (Ridley and Hall, 1992, 1994). EGF-, PDGF-, and insulin-dependent actin polymerization in membrane structures such as ruffles 
and lamellipodia requires Rac1 (Ridley et al., 1992; Nobes and Hall, 1995), whereas bradykinin-stimulated filopodia formation requires $\mathrm{Cdc} 42 \mathrm{Hs}$ (Kozma et al., 1995; Nobes and Hall, 1995). Recent reports have provided evidence that Rho proteins might also link the reorganization of actin cytoskeleton and vesicular transport between organelles: expression of active RhoD modifies early endosome dynamics and distribution, but also activates the formation of filopodiallike structures at the cell periphery and causes a loss of stress fibers and the disassembly of focal adhesion complexes (Murphy et al., 1996), whereas Rac1 and RhoA control transferrin receptor-mediated endocytosis through the regulation of clathrin-coated vesicle formation (Lamaze et al., 1996).

In addition to their role in cell morphology, Rho proteins are involved in normal and pathological cell proliferation. Constitutively active RhoA, Rac1, or Cdc42Hs triggers entry of quiescent fibroblasts into S phase (Olson et al., 1995; Hirai et al., 1997) and promote the activation of the serum response factor, a transcription factor that regulates the expression of many growth factor-regulated genes (Hill et al., 1995). Rho members are also involved in Ras-mediated transformation (Khosravi-Far et al., 1995, 1996; Qiu et al., 1995a,b, 1997; Roux et al., 1997) and delineate distinct pathways that cooperate to transform NIH 3T3 cells (Roux et al., 1997). Furthermore, Rac1 activation has been shown to play a role in metastasis (Habets et al., 1994; Michiels et al., 1995). Several Rho proteins have been implicated in programmed cell death: RhoA promotes cell apoptosis of NIH 3T3 cells through ceramide production (Esteve et al., 1995; Jimenez et al., 1995), and activity of RhoA, Rac1, and Cdc42Hs proteins is required for Fas ligand-mediated apoptosis in T cells (Gulbins et al., 1996; Moorman et al., 1996; Brenner et al., 1997). The role of Rho proteins in the apoptotic process might be linked to the activation of stress kinase pathways [including c-jun $\mathrm{N}$-terminal or stress-activated protein kinase and RK/p38 kinases] (Coso et al., 1995; Minden et al., 1995; Teramoto et al., 1996; Roux et al., 1997).

Although the Rho members characterized thus far control specific aspects of the cellular metabolism, in some instances, activation of distinct Rho members leads to similar phenotypes. This might result from activation of common downstream effector proteins such as the p21-activated kinase $\mathrm{p} 65^{\mathrm{PAK}}$ or the WASP, which both bind Rac1 and Cdc42Hs (Manser et al., 1994; Martin et al., 1995; Aspenstrom et al., 1996; Kolluri et al., 1996; Lim et al., 1996; Symons et al., 1996). Similarly, PRK2 and citron have both been reported to bind Rac1 and RhoA (Madaule et al., 1995; Vincent and Settleman, 1997), whereas p160 ROCK interacts with Cdc42Hs and RhoA (Fujisawa et al., 1996; Ishizaki et al., 1996; Leung et al., 1996). Alternatively, Rho GTPases might produce similar phenotypes through coordinated regulation of a cascade of activations. Sequential formation of polymerized actin structures has been described in microinjected serum-starved Swiss $3 \mathrm{~T} 3$ cells, indicating that activation of $\mathrm{Cdc} 42 \mathrm{Hs}$ activates Rac1, which in turn activates RhoA (Ridley and Hall, 1992; Ridley et al., 1992; Nobes and Hall, 1995; Tapon and Hall, 1997). However, in other cell lines and under different physiological conditions, Cdc42Hs and Rac1 compete or even antagonize RhoA-mediated activities (Lim et al., 1996; Leeuwen et al., 1997).

RhoG protein shares $72 \%$ and $62 \%$ sequence identity with Rac1 and Cdc42Hs, respectively, and was first characterized as expressed from a growth-stimulated gene (Vincent et al., 1992; Le Gallic and Fort, 1997). We recently reported that RhoG might share similar properties with Rac1 in the control of cell contact inhibition and transformation (Roux et al., 1997). To address the functional relationships between RhoG and the other Rho members, we analyzed changes in cell morphology, polymerized actin structures, and membrane surface organization in RhoG-expressing rodent cells using confocal and scanning electron microscopy. Here, we show that expression of active RhoG elicited both Rac1- and Cdc42Hs-like events, including the formation of membrane ruffles, lamellipodia, filopodia, and microvilli. These effects were not mediated through a direct interaction with Rac1 or Cdc42Hs target proteins, but rather required the activity of endogenous Rac1 and Cdc42Hs. The RhoG-induced phenotype was specifically inhibited upon microtubule depolymerization, whereas under these conditions, Rac1and $\mathrm{Cdc} 42 \mathrm{Hs}$-dependent cytoskeletal reorganization could still be stimulated by PDGF and bradykinin.

\section{MATERIALS AND METHODS}

\section{DNA Constructs}

Chimeras between enhanced green fluorescent protein (GFP) and RhoG were obtained by insertion of the RhoG G12V and T17N mutant ORFs (Roux et al., 1997) in pEGFP-C1 (Clontech, Palo Alto, CA). Hemagglutinin (HA)-epitope-tagged RhoG, Rac1, and Cdc42Hs ORFs inserts were transferred into the pLXSN retroviral vector for infection. Constructs expressing MYC epitope-tagged mutant Rac1 and Cdc42Hs proteins (Dutartre et al., 1996) were kindly provided by P. Chavrier.

\section{Two-Hybrid Interaction}

ORFs encoding constitutively active RhoG, Rac1, and Cdc42Hs and RhoA GTPases were subcloned in pBTM116 and used to transform the yeast L40 strain (MATa trp1 leu2 his3 lys2::lexA-his3 ura3::lexA-LacZ). PAK-1 and POR1 ORFs were obtained by PCR amplification and subcloned in pGAD1318. The WASP insert (encoding aa 201-321) was swapped from pGexKG to pGAD1318. pGAD1318-Kinectin (clone 66, encoding aa 677-913) was isolated from an interaction screen with RhoG. pGexK-WASP and pActIIROCK (encoding aa 888-1030) were kindly provided by A. Hall. L40 and AMR70 strains were mated and diploids were selected on drop-out medium lacking leucine and tryptophane. Diploids were 
patched on Whatmann filters and processed for $\beta$-galactosidase activity as described (Chardin et al., 1993).

\section{Cell Culture, Transfection, and Microinjection}

Rat embryo fibroblasts (REF-52) or mouse Swiss 3T3 fibroblasts were cultured at $37^{\circ} \mathrm{C}$ in the presence of $5 \% \mathrm{CO}_{2}$ in DMEM supplemented with $10 \%$ FCS. Cells were plated on 18-mm diameter glass coverslips $16-24 \mathrm{~h}$ before transfection or microinjection. Cells were transfected using the lipofectamine method $(0.5-1 \mu \mathrm{g}$ of plasmid DNA per well containing three glass coverslips), as described by the supplier (Life Technologies, Gaithersburg. MD). Four hours after the transfection, the medium was replaced by plain DMEM or supplemented with 10\% FCS. Expressing cells were observed under immunofluorescence microscopy $8-24 \mathrm{~h}$ after transfection. Alternately, plasmid DNA $\left(0.1 \mathrm{mg} \cdot \mathrm{ml}^{-1}\right)$ was microinjected into the cell nucleus. At various times after microinjection, cells were fixed and processed for immunohistochemistry.

\section{Subcellular Fractionation, Protein Separation, and Immunoblotting}

Transiently transfected Cos 7 cells were washed twice with PBS and then lysed in cold hypotonic buffer containing $10 \mathrm{mM}$ Tris- $\mathrm{HCl}(\mathrm{pH}$ $7.5), 5 \mathrm{mM} \mathrm{MgCl}, 1 \mathrm{mM}$ DTT, and $1 \mathrm{mM}$ PMSF. Cells were lysed by quick freezing in liquid nitrogen and rapid thawing at $65^{\circ} \mathrm{C}$. Under these conditions, $>95 \%$ of cells were lysed, as monitored by microscopy. Cell extracts were centrifuged $\left(600 \times g\right.$ for $5 \mathrm{~min}$ at $\left.4^{\circ} \mathrm{C}\right)$ to pellet nuclei and nuclei-associated structures (P1). Supernatants were ultracentrifuged $(100,000 \times g$ for $45 \mathrm{~min})$ to separate free cytoplasmic membranes (P100) and cytosolic proteins (S100). Samples were fractionated on a $15 \%$ SDS-polyacrylamide gel and electrotransferred to nitrocellulose membranes as described by the supplier (Millipore, Bedford, MA). Membranes were blocked in PBS containing $8 \%$ (wt/vol) powdered milk, followed by incubation with 12CA5 hybridoma culture supernatant and horseradish peroxidase-conjugated sheep anti-mouse antibodies (1:2000 dilution, Amersham, Arlington Heights, IL). Signals were revealed using a chemiluminescent reagent (New England Nuclear, Boston, MA).

\section{Infection Procedures}

G418-resistant GP+E-86 clones expressing constitutively activated or dominant negative forms of RhoG, Rac1, or Cdc42Hs were grown to collect retrovirus-containing cell-free supernatants (Roux et al., 1997). Infection in the absence of selection was performed on exponentially growing REF-52 or Swiss 3T3 cells (seeded at $5 \times 10^{5}$ cells per $60-\mathrm{mm}$ diameter plate) using $5 \mathrm{ml}$ of viral supernatant $\left(10^{5}\right.$ to $5 \times 10^{5}$ colony forming units per $\mathrm{ml}$ ). A first round of infection was performed directly during cell recovery after trypsinization, followed by a second round 16-24 h later. Cells were incubated for $20 \mathrm{~h}$ and then processed for scanning electron microscopy (SEM). Alternatively, infected cells were grown to confluence and then split and seeded into selective medium $\left(1 \mathrm{mg} \cdot \mathrm{ml}^{-1} \mathrm{G} 418\right)$. After $10 \mathrm{~d}$ of selection, resistant colonies were collected, tested by indirect immunofluorescence using the anti-MYC mAb (clone 9E10) and processed for SEM. For double infection experiments, stable transformants were transiently infected as described above and processed for SEM.

\section{Immunofluorescence}

At different times after microinjection or transfection, cells were fixed for $5 \mathrm{~min}$ in $3.7 \%$ formalin (in PBS) followed by a 2-min permeabilization with $0.1 \%$ Triton X-100 (in PBS) and incubation in PBS containing $0.1 \%$ BSA. Alternatively, cells were fixed for $20 \mathrm{~min}$ in a microtubule-protecting buffer containing 3\% formalin, $0.05 \%$ glutaraldehyde, $0.05 \%$ Triton $X-100$ in $60 \mathrm{mM}$ piperazine- $N, N^{\prime}$ bis(2-ethanesulfonic acid), 25 mM HEPES (pH 6.9), 10 mM EGTA, and $10 \mathrm{mM} \mathrm{MgCl}$ followed by a 2-min permeabilization with $0.2 \%$ Triton $\mathrm{X}-100$ in $50 \mathrm{mM}$ Tris- $\mathrm{HCl}(\mathrm{pH} \mathrm{7.5)}$ and $150 \mathrm{mM} \mathrm{NaCl}$. Expression of GFP-tagged proteins was directly visualized, whereas expression of MYC epitope-tagged proteins was visualized after a 60-min incubation with the 9E10 anti-MYC mAb (gift from C. Lambert and D. Mathieu, Montpellier, France) (1:2 dilution in PBS/ BSA), followed by incubation with affinity-purified fluorescein-conjugated goat anti-mouse antibody (Cappel-Organon Technika Fresnes, France) (1:40 dilution). Cells were simultaneously stained for F-actin, phospho-tyrosine epitopes, and RhoG using rhodamineconjugated phalloidin $\left(0.5 \mathrm{U} \cdot \mathrm{ml}^{-1}\right.$, Molecular Probes, Eugene, OR), the $4 \mathrm{G} 10 \mathrm{mAb}$ (a gift from M. Martin and P. Mangeat, Montpellier, France), and a rat antiserum raised against RhoG protein purified from baculovirus-infected Sf9 cells (batch R2), respectively. For simultaneous detection of GFP-tagged proteins, MYC-tagged proteins, and F-actin, anti-MYC 9E10 staining was detected at a 445-nm wavelength using 7-amino-4-methylcoumarin-3-acetic acid-conjugated donkey anti-mouse IgG (1:50 dilution, Jackson ImmunoResearch Labs, West Grove, PA). Cells were washed in PBS, mounted in Mowviol (Aldrich, Milwaukee, WI) and observed under a laser scanning confocal microscope (MRC-1024, Bio-Rad Laboratories, Richmond, CA) or a DMR Leica microscope using a $63 \times$ planapochromat lens. For all experiments, at least 100 transfected cells were examined. Images were recorded using a kappa camera, transferred to Adobe Photoshop, assembled in Canvas, and printed out on a Kodak ColorEase thermal sublimation printer.

\section{Confocal Laser Scanning Microscopy}

Dual-channel confocal laser scanning microscopy was performed using the Bio-Rad MRC 1024 confocal laser scanning microscope equipped with an argon/krypton ion laser. For all experiments, at least 100 transfected cells were examined. Images were collected sequentially to avoid cross-contamination between the fluorochromes. Series of optical sections through the cell were collected and projected onto a single image plane. Images were processed as described above.

\section{SEM}

Transiently or stably infected REF-52 or Swiss 3T3 cells were grown on glass coverslips and then fixed in $0.1 \mathrm{M}$ sodium cacodylate $(\mathrm{pH}$ 7.2) containing $2 \%$ glutaraldehyde and $0.1 \mathrm{M}$ sucrose for at least $1 \mathrm{~h}$ and processed as described (Brunk et al., 1981). Samples were observed using a Hitachi S4000 scanning microscope at $15 \mathrm{kV}$. For all experiments, at least 50 cells were examined. Images were processed as described above.

\section{RESULTS}

\section{Localization of Endogenous and Chimeric GFP RhoG Proteins}

GFP-RhoG fusion proteins were constructed in the mammalian expression vector pEGFP-C1, fusing the enhanced GFP coding sequence upstream of mutant RhoG ORFs. Two mutant RhoG proteins were expressed: one carrying the G12V substitution, leading to a constitutively active protein, and the other one carrying the T17N substitution, acting as an inhibitor of the endogenous protein (Roux et al., 1997). To assess the level of expression and subcellular distribution of the chimeras, REF-52 cells were transfected with GFPRhoGV12 or GFP-RhoGN17, and fixed cells were observed using fluorescence microscopy (Figure 1A). In all transfected cells, the GFP-RhoGV12 fusion protein 
A
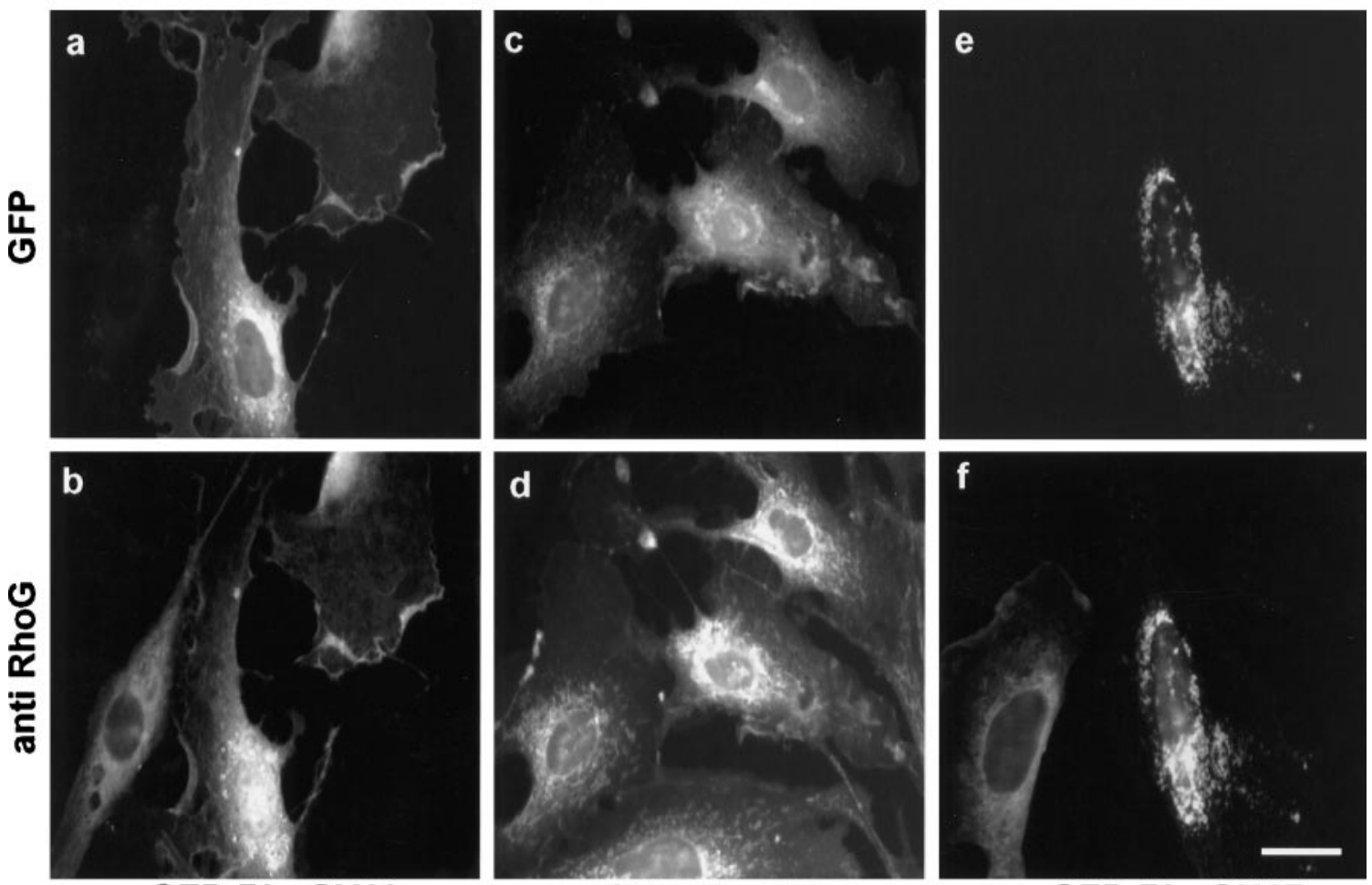

GFP-RhoGV12

GFP-RhoGV12

GFP-RhoGN17

B

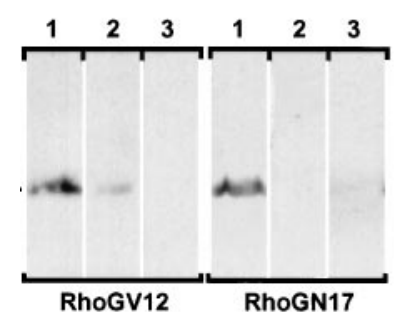

Figure 1. Subcellular localization of GFP-tagged and endogenous RhoG proteins. (A) Exponentially growing REF-52 cells were transfected with plasmids encoding GFP-RhoGV12 (panels a-d) or GFP-RhoGN17 (panels e and f) proteins. Cells were fixed $18 \mathrm{~h}$ after transfection in 3.7\% formalin (panels $a, b, e$, and f) or in a microtubule-protecting buffer (panels c and d). Cells were monitored for GFP fluorescence (panels a, c, and e) and endogenous RhoG distribution using a rat polyclonal antibody (panels $\mathrm{b}, \mathrm{d}$, and $\mathrm{f}$ ). Bar, $10 \mu \mathrm{m}$. For each panel, cells shown are representative of more than 100 observed cells. (B) Cos7 cells were transfected with constructs expressing HA epitope-tagged RhoGV12 or RhoGN17 proteins. Cells were lysed in the absence of detergent, and nuclei and associated membranes were pelleted at low-speed centrifugation (lanes 1). The supernatant was then submitted to ultracentrifugation, resulting in the isolation of P100 plasma membranes (lanes 2) and S100 cytosolic extracts (lanes 3). Proteins from each fraction, corresponding to identical cell number, were analyzed by Western blotting and revealed with the anti-HA 12CA5 antibody.

exhibited a punctuated distribution throughout the cytoplasm, with a marked concentration in the perinuclear region (panel a) and a minor fraction at the plasma membrane. As a control, GFP protein expressed from $\mathrm{pEGFP-C1}$ showed a diffuse cytoplasmic staining. Immunodetection of RhoG protein was also performed using a rat polyclonal antibody raised against a recombinant RhoG protein produced from baculovirus infected-Sf9 cells (see MATERIALS AND METHODS). As observed in panel $b$, the distribution of endogenous RhoG protein showed a staining very similar to the GFP fluorescence observed with the chimeric RhoG protein (panel a). This colocalization was further investigated using a microtubule-protecting fixation buffer instead of formalin (panels c and d). Under these conditions, both endogenous RhoG and the chimeric RhoG protein exhibited an overall distribution similar to that shown in panels a and b, with the perinuclear staining appearing as well-defined short tubular structures, reminiscent of endoplasmic reticulum staining. In contrast, the GFP-RhoGN17 chimera exhibited a different distribution characterized by larger dots concentrated at the perinuclear region, suggesting that only the GTP-bound RhoGV12 protein could distribute to the plasma membrane and throughout the cytoplasm (panel e). Interestingly, the distribution of the endogenous RhoG protein was modified in RhoGN17-expressing cells and colocalized with the GFP chimera (panel f), strongly suggesting that the N17 protein impairs the activity of the endogenous protein. Further analysis of the compartimentalization of RhoGV12 and RhoGN17 proteins 
was performed in Cos7 cells transfected with constructs expressing HA epitope-tagged proteins. Cells were lysed in the absence of detergent, and cell extracts were fractionated by differential centrifugation, resulting in the separation of nuclei and associated membranes, plasma membranes, and cytosol (Figure 1B). Western blot analysis showed that both mutated proteins were found predominantly associated with nuclei-bound membranes (lanes 1), a minor fraction of RhoGV12, but not RhoGN17, cofractionated with the plasma membranes (lanes 2). No protein could be detected in the cytosolic fraction (lanes $3)$. These data were consistent with the fluorescence observations.

\section{RhoGV12 Induces the Formation of Ruffles, Lamellipodia, Microvilli, and Filopodia Followed by Partial Disassembly of Actin Stress Fibers}

To examine the overall effect of RhoG activation, plasmid DNA encoding GFP-RhoGV12 was microinjected into the nucleus of quiescent or exponentially growing REF-52 fibroblasts, and modifications in polymerized actin-containing structures were examined by staining with rhodamine-labeled phalloidin (Figure 2A). Six hours after microinjection, GFP-RhoGV12 protein was detected throughout the cytoplasm, with intense staining at the cell periphery and onto the dorsal membrane (panel a). F-actin costaining (panel b) showed that GFP-RhoGV12 colocalized with ruffles and lamellipodia (arrowed as R and L). Eight to $12 \mathrm{~h}$ after microinjection, GFP fluorescence was found mainly around the nucleus and at the cell periphery (panels c and e). F-actin costaining (panels $\mathrm{d}$ and $\mathrm{f}$ ) still revealed peripheral lamellipodia and a reduced number of ruffles (arrowed as R and L) as well as the emission of small radial protrusions (open arrows). Protrusions and retraction fibers were discriminated using timelapse microscopy. Concomitantly with the appearance of the membrane structures, actin stress fibers were progressively reduced (panels $\mathrm{b}-\mathrm{f}$ ) and were no longer detectable by $14 \mathrm{~h}$ after microinjection in $50-70 \%$ of the expressing cells (panels $\mathrm{f}$ and $\mathrm{h}$ ). Cells exhibited an altered morphology, with a retracted cell body and large protrusions ending with lamellipodial- and filopodial-like structures. This phenotypic change was accompanied by the formation of focal complexes at the cell periphery, as revealed by anti-phospho-tyrosine staining (Figure 2B, panel b), whereas control cells exhibited focal adhesions which appeared as larger patches distributed on the basal membrane (panel c). Similar redistribution of focal adhesions has been previously reported upon expression of activated Rac1V12 protein (Nobes et al., 1995).

The effects of RhoG were also examined in Swiss $3 \mathrm{~T} 3$ cells, which have been extensively used to study the actin filament network (Ridley and Hall, 1992)
(Figure 2C). Because these cells are not suitable for nuclear microinjection, GFP-RhoGV12 expression was achieved by DNA transfection. As in REF-52 cells, GFP-RhoGV12 exhibited a distribution throughout the cytoplasm with local accumulation around the nucleus and in actin-containing structures (panels a and c). Eighteen hours after transfection (panels c and d), cells exhibited changes in their morphology similar to those observed in panel $g$ of Figure 2A, with a retraction of the cell body and extrusion of long cytoplasmic extensions, containing both central and distal protrusions and lamellipodia. Expression of GFP protein alone had no effect on actin filament structure or cell shape.

\section{RhoGV12 Induces Changes in Polymerized Actin Structures and Cell Morphology Similar to Those Mediated by Rac1 and Cdc42Hs}

In fibroblastic cells, Rac1 mediates local actin polymerization leading to the formation of ruffles and lamellipodia (Ridley et al., 1992), whereas Cdc42Hs induces the formation of peripheral-actin microspikes, filopodia, and reduction in the number of stress fibers (Kozma et al., 1995; Nobes and Hall, 1995). Since the overall effects of GFP-RhoGV12 expression appeared similar to those induced by Rac1 and Cdc42Hs, we more precisely compared the modifications in actin and plasma membrane structures resulting from the introduction of each constitutively active GTPase using rhodamine-labeled phalloidin staining and scanning electron microscopy, respectively. GFPRhoGV12, MYC-Rac1V12, or MYC-Cdc42HsV12 proteins were expressed by transfection of Swiss 3T3 cells, and cells were fixed $18 \mathrm{~h}$ later (Figure 3). Although the overall cellular morphology of RhoGV12-expressing cells appeared very similar to the one obtained after Rac1V12 expression (compare panels $a$ and $b$, and panels $\mathrm{c}$ and $\mathrm{d}$ ), additional radial extensions containing F-Actin were detected in RhoGV12-cells (panels a and $b$ ). Cdc42HsV12-expressing cells produced similar extensions around their periphery (panels e and f), but exhibited a more regular rounded shape. In addition, $60 \%$ of Rac1V12- and Cdc42HsV12-expressing cells showed disassembly of actin stress fibers. These data indicate that RhoGV12 and Rac1V12 promote similar cell shape reorganization and formation of F-actin-containing structures and also show partial overlapping effects of RhoGV12 and Cdc42HsV12.

We further analyzed structural changes on cell dorsal membranes using SEM. Because SEM cannot discriminate between transfected and nontransfected cells, REF-52 and Swiss 3T3 fibroblastic cells were retrovirally infected instead of transfected to allow ectopic expression in more than $90 \%$ of the cells, as monitored by immunocytochemistry. After fixation 
A

$6 h$
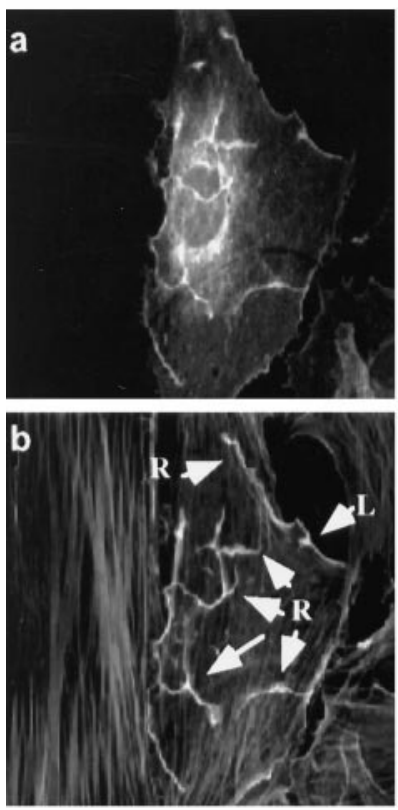

$8 \mathrm{~h}$
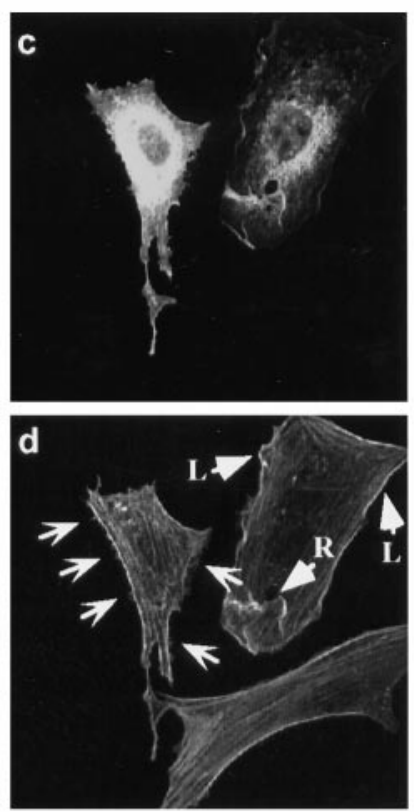

$12 \mathrm{~h}$
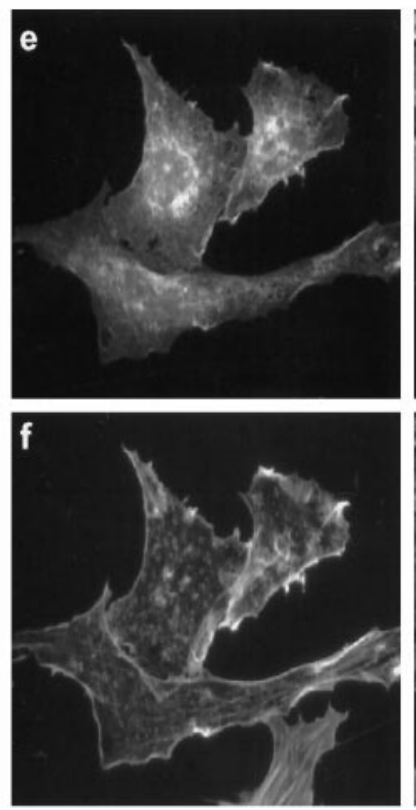

$18 h$
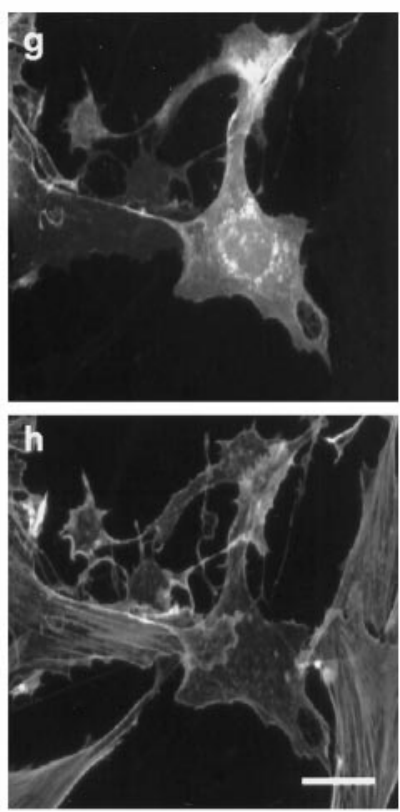

B
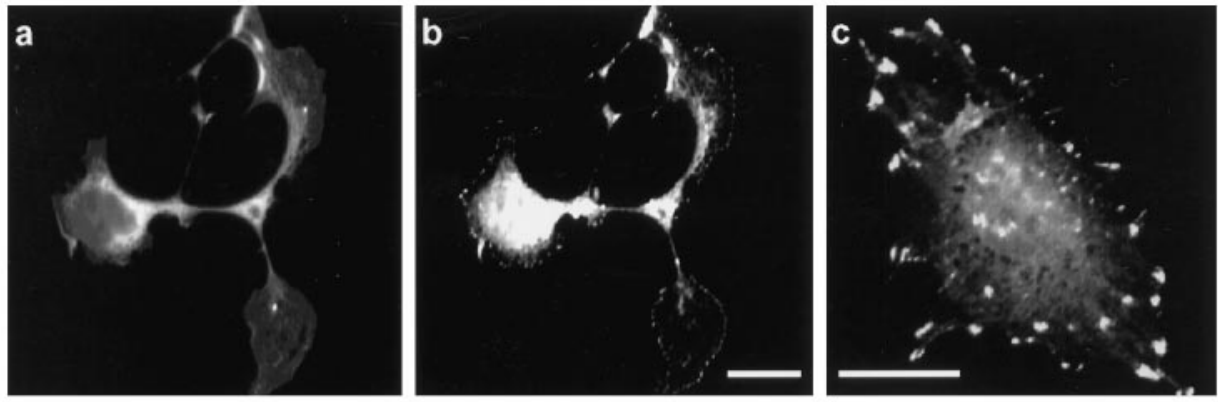

C

$7 \mathrm{~h}$

$18 \mathrm{~h}$
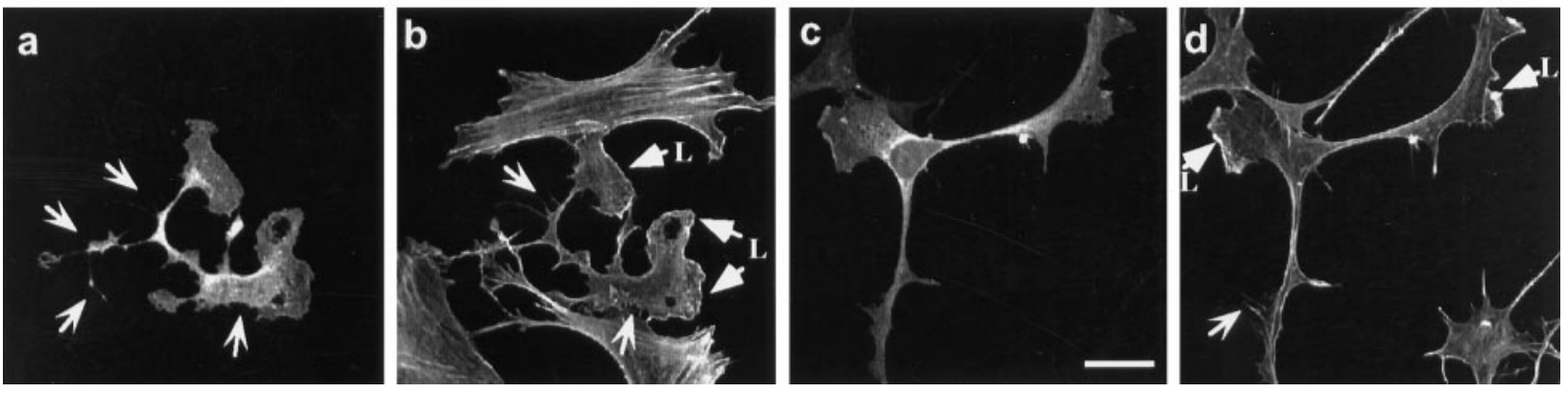

Figure 2. Actin reorganization induced by GFP-RhoGV12 protein expression in REF-52 and Swiss 3 T3 cells. (A) Quiescent REF-52 cells were microinjected with plasmids encoding GFP-RhoGV12 protein. Microinjected cells were fixed after different periods of time and then stained with rhodamine-labeled phalloidin. Cells were observed under confocal laser scanning microscopy for GFP activity (panels a, c, e, and g) and for filamentous actin distribution (panels $\mathrm{b}, \mathrm{d}, \mathrm{f}$, and $\mathrm{h}$ ). Arrows designated with an $\mathrm{R}$ and $\mathrm{L}$ indicate dorsal ruffles and lamellipodia, respectively, whereas open arrows indicate filopodia. Bar, $10 \mu \mathrm{m}$. (B) Exponentially growing Swiss 3T3 cells were transfected with constructs expressing GFP-RhoGV12 protein and serum starved for $14 \mathrm{~h}$. Cells were fixed and monitored for GFP activity (panel a) and 
Figure 3. Comparison of changes in cell morphology and actin distribution induced by RhoGV12, Rac1V12, and Cdc42HsV12 proteins. Swiss 3T3 fibroblasts were transfected with constructs expressing GFP-RhoGV12 (panels a and b), MYC epitope-tagged Rac1V12 (panels $\mathrm{c}$ and d), or MYC epitope-tagged Cdc42HsV12 (panels e and f) proteins. Cells were fixed $18 \mathrm{~h}$ after transfection and processed for GFP activity (panel a), MYC epitope detection (panels $\mathrm{c}$ and $\mathrm{e}$ ), and filamentous actin detection (panels $\mathrm{b}, \mathrm{d}$, and f). Bar, $10 \mu \mathrm{m}$. For each panel, cells shown are representative of more than 100 observed cells.
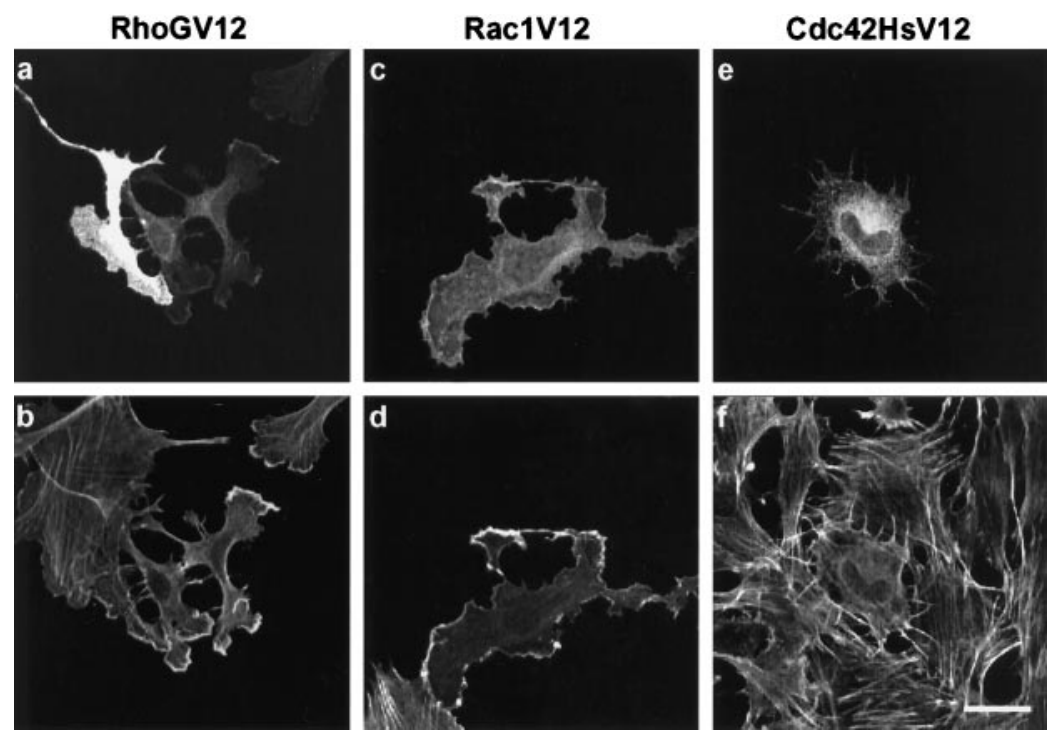

and processing for SEM, cells were analyzed on a Hitachi S4000 scanning microscope (Figure 4). Exponentially growing control cells (i.e., noninfected cells or cells infected with wild-type virions) presented a smooth dorsal membrane and low activity at the edge of the cell (panels a). Expression of Rac1V12 induced the formation of large lamellipodia at the cell periphery, whereas the dorsal membrane maintained a smooth appearance (panels b). Expression of Cdc42HsV12 promoted the formation of filopodial extensions (panels c) and elicited a high density of microvilli on the cell surface (shown at higher magnification in the insert of panel c for REF-52 fibroblasts). Expression of RhoGV12 led to a mixed phenotype, consisting of large lamellipodia and filopodia (panels d) and a high density of microvilli (shown at higher magnification in the insert of panel d for REF-52 fibroblasts). Similar microvilli were recently reported to occur in RhoA-expressing cells (Shaw et al., 1998). Taken together, these data indicate that RhoGV12 promotes actin reorganization and morphological modifications similar to those elicited by the combination of Rac1V12 and Cdc42HsV12.

Figure 2 (cont). phosphotyrosine-containing epitopes (panels b and c). (C) Exponentially growing Swiss 3T3 cells were transfected with constructs expressing GFP-RhoGV12 protein. Cells were either fixed $7 \mathrm{~h}$ (panels a and b) or $18 \mathrm{~h}$ (panels c and d) after transfection and stained with rhodamine-labeled phalloidin. Cells were monitored for GFP activity (panels a and c) and filamentous actin (panels $\mathrm{b}$ and $\mathrm{d}$ ). Arrows designated with an $\mathrm{L}$ indicate lamellipodia whereas open arrows indicate filopodia. For each panel, cells shown are representative of more than 100 observed cells.
RhoGV12-dependent Cytoskeletal Reorganization Is Mediated through Endogenous Rac1 and Cdc42Hs Activity

To address the functional relationship among RhoG, Rac1, and Cdc42Hs, we first examined whether RhoG might produce its structural effects by a direct activation of the downstream target proteins of Rac1 and Cdc42Hs. We used the yeast two-hybrid interaction assay to test the ability of RhoGV12 to bind p65 PAK-1, a kinase activated by Rac1 and Cdc42Hs (Manser et al., 1994); POR-1, a potential effector of Rac1 involved in membrane ruffling (Joneson et al., 1996; Van Aelst et al., 1996); WASP, a Cdc42Hs target whose inactivation leads to dysregulated membrane structures associated with the WASP (Symons et al., 1996); and p160 ROCK (Leung et al., 1996), a RhoA-interacting kinase that controls the formation of stress fibers. As a positive control, we used kinectin, a kinesin receptor anchored in intracytoplasmic membranes, which had been isolated from a yeast interaction trap with activated RhoAV14 (Hotta et al., 1996) and RhoGV12 (our unpublished data). Diploid yeast strains expressing each pair of hybrid proteins were processed for $\beta$-galactosidase activity (Figure 5). Strains expressing RhoGV12 fusion did not reveal any interaction with full-length PAK-1 and POR1 nor with the interacting regions of WASP (aa 201-321) or p160 ROCK (aa 888-1030), whereas an interaction with kinectin (aa 677-913) was readily detected. The expected control pattern was observed, with Rac1V12 interacting with PAK-1, POR1, and kinectin, Cdc42HsV12 binding to POR1 and WASP, and RhoAV14 associating with p160 ROCK and kinectin. These data suggest that the morphological effects mediated by RhoGV12 were not due to direct interactions with targets of Rho 
A
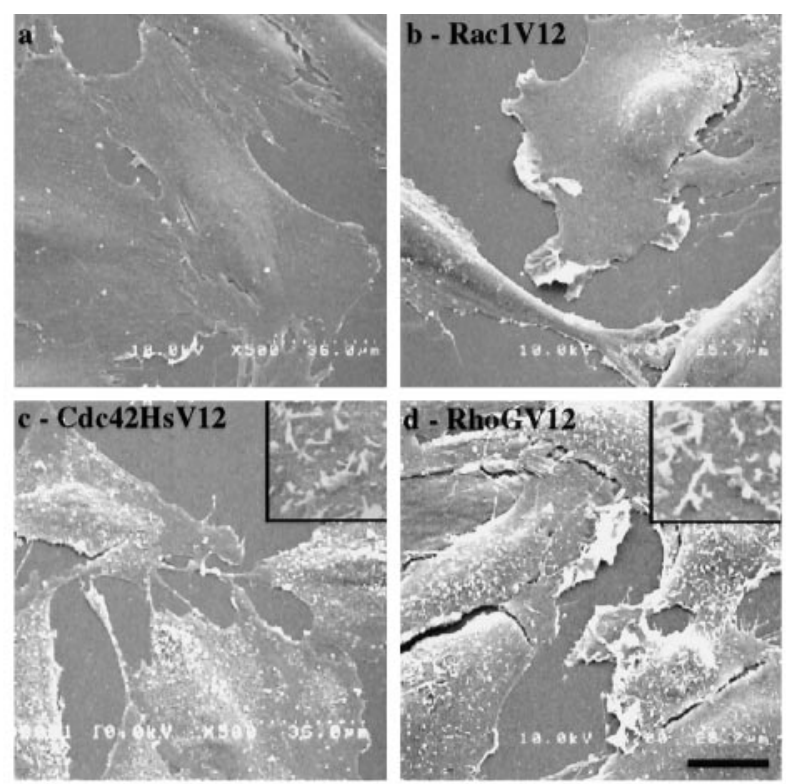

REF-52
B
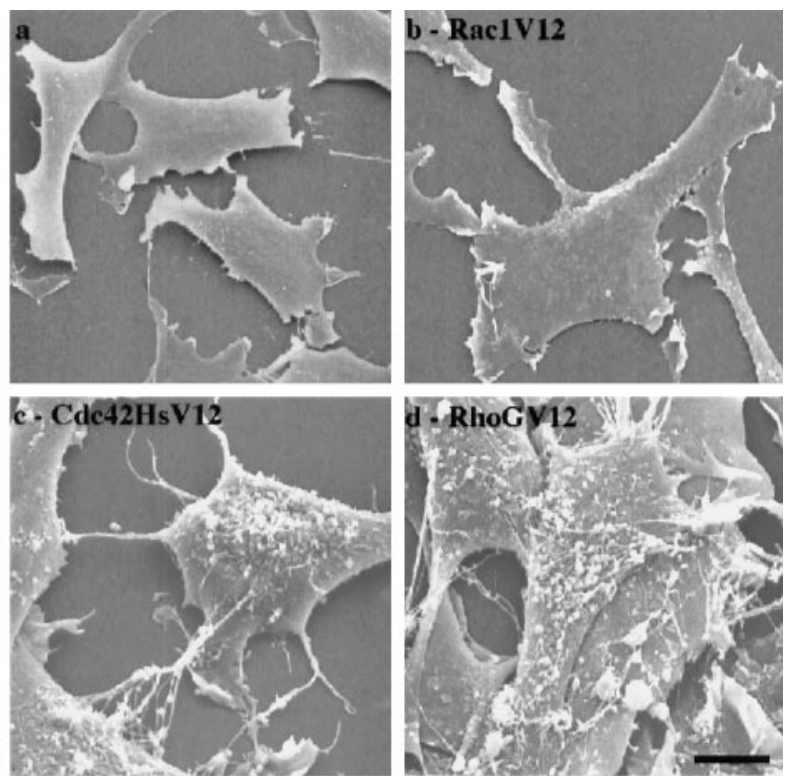

Swiss 3 T3

Figure 4. Comparison of plasma membrane modifications induced by RhoGV12, Rac1V12, or Cdc42HsV12 proteins. REF-52 (A) and Swiss 3T3 (B) fibroblasts were infected with retroviruses encoding activated Rho GTPases and processed for SEM analysis. Shown are scanning electron micrographs of control cells (panels a) and cells expressing Rac1V12 protein (panels b), Cdc42HsV12 protein (panels c), or RhoGV12 protein (panels d). Higher magnification is shown in the inserts of panels c and d (in A). Bar, $10 \mu \mathrm{m}$. For each panel, cells shown are representative of more than 50 scanned cells.

family proteins known to control actin polymerization.

We next examined whether RhoGV12 might require endogenous Rac1 and Cdc42Hs activity to produce rearrangements in polymerized actin and cell morphology. For this purpose, we coexpressed RhoGV12 in Swiss 3T3 cells with dominant negative forms of either Rac1 (Rac1N17) or Cdc42Hs

\section{PAK POR1 WASP ROCK KINECTIN}

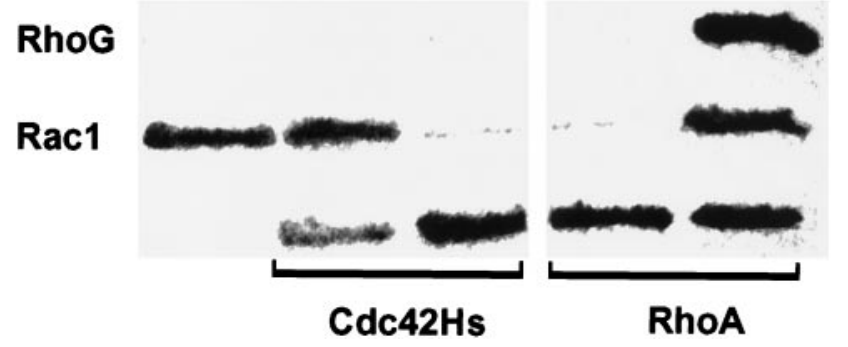

Figure 5. Interaction between RhoG and effectors of the Rho family in the yeast two-hybrid system. AMR70 yeast strains expressing full-length PAK-1 and POR1 or Rho-interacting fragments of WASP (aa 201-321), ROCK (aa 888-1030), and kinectin (aa 677-913) fused to Gal4-activating domain were mated with L40 strains expressing RhoGV12, RhoAV14, Rac1V12, or Cdc42HsV12 mutated in their CAAX boxes and fused to the LexA DNA-binding domain. Diploids were patched on Whatman paper and processed for $\beta$-galactosidase activity for $3 \mathrm{~h}$ at $30^{\circ} \mathrm{C}$.
(Cdc42HsN17). Effects on F-actin distribution and cell morphology were monitored by immunocytochemistry using rhodamine-labeled phalloidin and SEM. A third emission wavelength at $445 \mathrm{~nm}$ was used to detect MYC-Rac1N17 or MYC-Cdc42HsN17 protein expression simultaneously with F-actin and GFP-RhoGV12 expression (Figure 6). Coexpression of GFP-RhoGV12 (panel a) and MYC-Rac1N17 (panel b) inhibited the emergence of lamellipodia and ruffles (compare with Figures 1 and 2), whereas the formation of filopodial extensions was still observed (panel c). Conversely, coexpression of MYCCdc42HsN17 (panel e) with GFP-RhoGV12 (panel d) prevented the formation of large protrusions and filopodia, but did not impair the formation of lamellipodia (panel f). Expression of either Rac1N17 (panel g) or Cdc42HsN17 (panel i) alone had no effect on cell morphology or actin distribution (panels $\mathrm{h}$ and j). Furthermore, coexpression of RhoGN17 did not impair Rac1V12-dependent lamellipodia nor Cdc42HsV12-dependent filopodia formation (our unpublished results). Similar results were observed in REF-52 cells.

We next examined the effects of Rac1N17 and Cdc42HsN17 expression on RhoGV12-dependent membrane reorganization using SEM (Figure 7). RhoGV12-expressing REF-52 cells were infected with retroviruses harboring either Rac1N17 or 

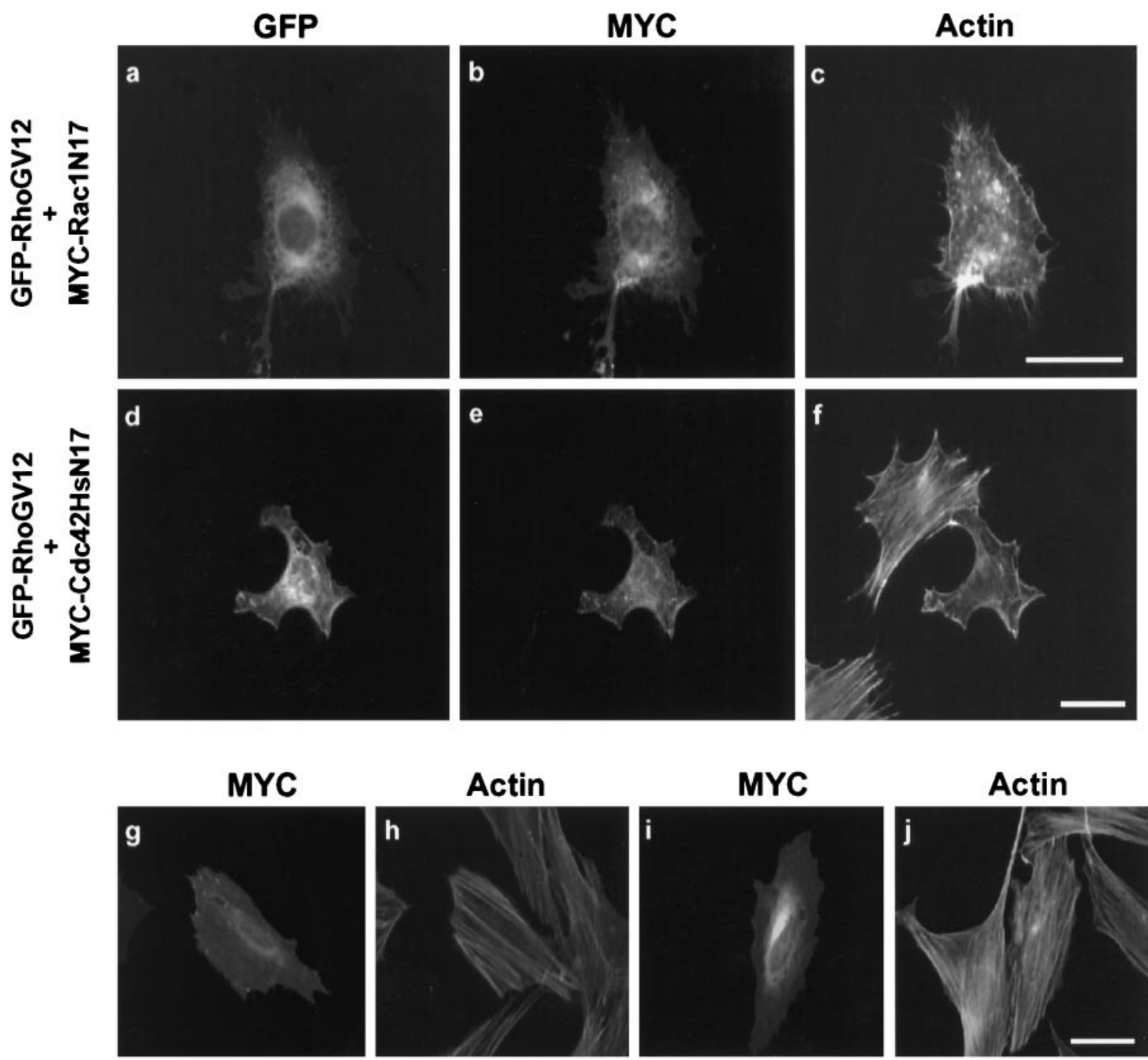

MYC-Rac1N17

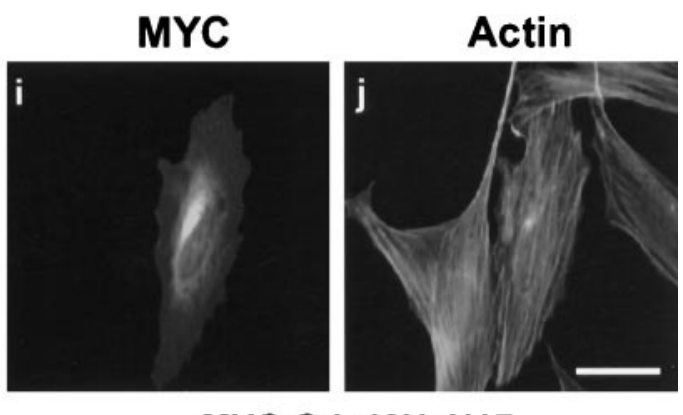

MYC-Cdc42HsN17

Figure 6. Effects of Rac1 and Cdc42Hs inhibition on RhoG-dependent actin reorganization. Swiss 3T3 cells were cotransfected with constructs expressing GFP-RhoGV12 and MYC epitope-tagged Rac1N17 (panels a-c) or Cdc42N17 proteins (panels d-f). As a control, cells were transfected only with constructs expressing MYC epitope-tagged Rac1N17 (panels g and h) or Cdc42HsN17 (panels i and j) proteins. Cells were fixed $18 \mathrm{~h}$ after transfection and incubated with the anti-MYC 9E10 mAb and rhodamine-labeled phalloidin. Cells were monitored for GFP expression (panels a and d), MYC-epitope detection (panels b, e, g, and i), and F-actin distribution (panels c, f, h, and j). Bar, $10 \mu \mathrm{m}$. For each panel, cells shown are representative of more than 100 observed cells.

Cdc42HsN17. RhoGV12 expression alone (panel a) induced the formation of lamellipodia and filopodia at the cell periphery and the formation of microvilli onto the dorsal membrane. Coexpression of RhoGV12 with Rac1N17 (panel b) led to a dramatic reduction of lamellipodia, whereas no change in filopodia or microvilli was detected. In contrast, coexpression of RhoGV12 with Cdc42HsN17 (panel c) induced a complete loss of filopodia and a large decrease in microvilli, whereas lamellipodia were still present at the cell periphery.
Taken together, these data indicate that the effects elicited by RhoGV12 require endogenous Rac1 activity for the formation of lamellipodia and Cdc42Hs activity for the formation of filopodia and microvilli but do not specifically act on the downstream targets of these two GTPases.

\section{RhoG Activity Is Not Required for PDGF- and} Bradykinin-dependent Rac1 and Cdc42Hs Activation Growth factors such as EGF, PDGF, and insulin have previously been reported to trigger Rac1-dependent 

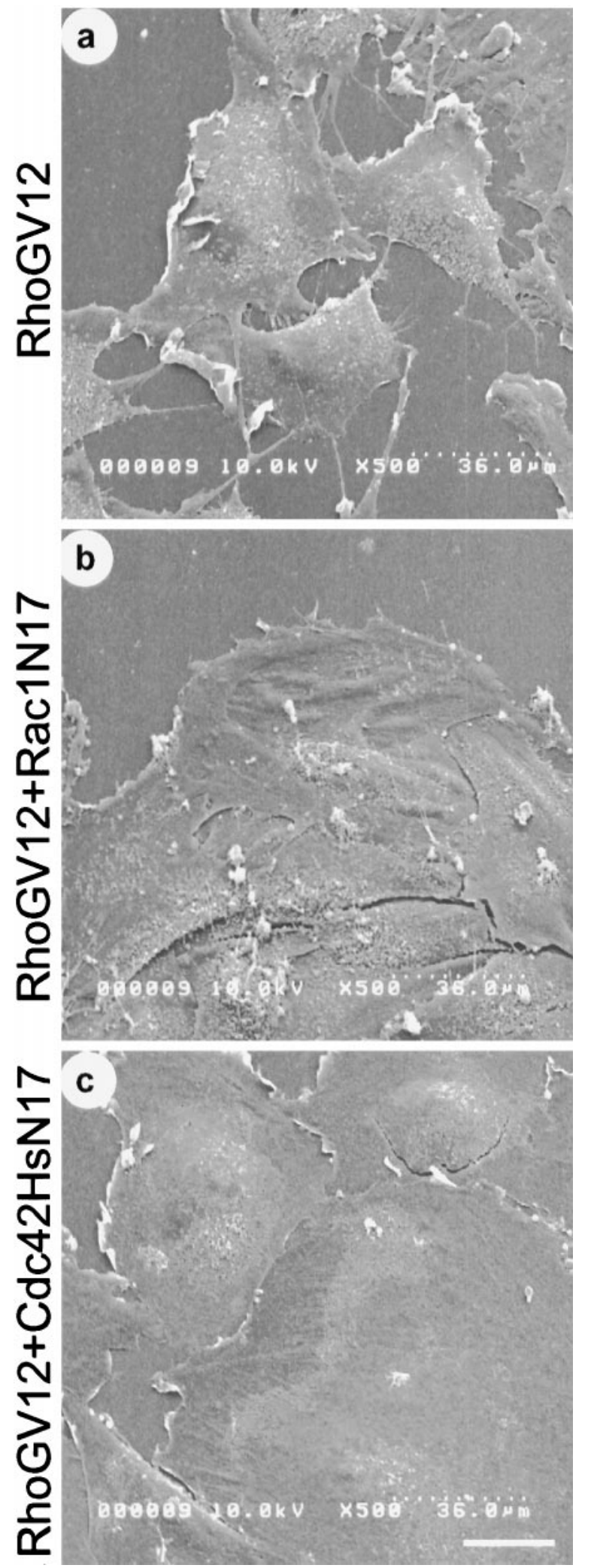

Figure 7. Effects of Rac1 and Cdc42Hs inhibition on RhoG-dependent plasma membrane modifications. RhoGV12-expressing REF-52

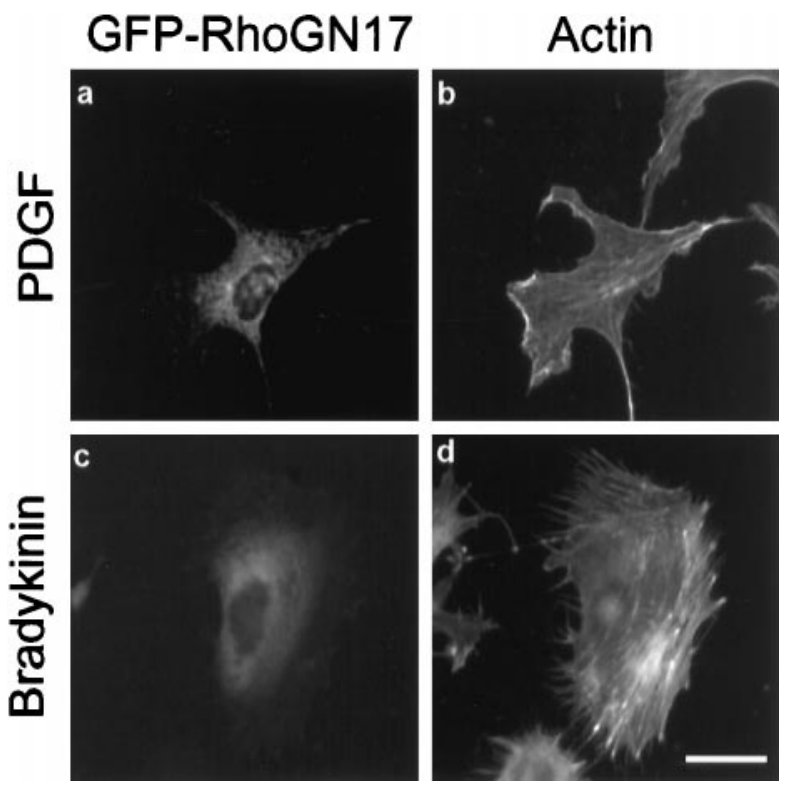

Figure 8. Effect of RhoGN17 expression on PDGF-induced lamellipodia and bradykinin-induced filopodia. Swiss 3T3 cells were transfected with constructs expressing GFP-RhoGN17 protein. Four hours after transfection, cells were serum starved for $20 \mathrm{~h}$. Cells were then treated for $10 \mathrm{~min}$ with $3 \mathrm{ng} \cdot \mathrm{ml}^{-1}$ PDGF (panels a and b) or $100 \mathrm{ng} \cdot \mathrm{ml}^{-1}$ bradykinin (panels $\mathrm{c}$ and $\mathrm{d}$ ), fixed, and monitored for GFP activity (panels a and c) or F-actin distribution (panels b and d). Bar, $10 \mu \mathrm{m}$. For each panel, cells shown are representative of more than 100 observed cells.

ruffling and lamellipodia formation (Ridley and Hall, 1992; Ridley et al., 1992), whereas stimulation with bradykinin has been shown to promote the formation of Cdc42Hs-dependent filopodia (Kozma et al., 1995). Since our preceding results strongly suggested that RhoG protein might activate Rac1- and Cdc42Hs-dependent pathways, we examined the possibility that RhoG might mediate the extracellular ligand signaling pathways leading to Rac1 and Cdc42Hs activation (Figure 8). Swiss 3T3 cells expressing RhoGN17 were serum starved for $24 \mathrm{~h}$ and stimulated with $3 \mathrm{ng} \cdot \mathrm{ml}^{-1}$ PDGF or $100 \mathrm{ng} \cdot \mathrm{ml}^{-1}$ bradykinin for $10 \mathrm{~min}$. Cells were then fixed and stained for protein expression and for F-actin distribution. In PDGF-stimulated cells (panel a), expression of RhoGN17 did not prevent the formation of ruffles and lamellipodia (panel b), whereas both structures were abolished upon Rac1N17 expression. Similarly, in bradykinin-treated

Figure 7 (cont). cells were submitted to two rounds of infection by retroviruses encoding either Rac1N17 or Cdc42HsN17 proteins. Cells were fixed $20 \mathrm{~h}$ after the second infection and processed for SEM. Shown are scanning electron micrographs of REF-52 cells expressing RhoGV12 protein alone (panel a) or in combination with Rac1N17 protein (panel b) or Cdc42HsN17 protein (panel c). Bar, 10 $\mu \mathrm{m}$. Cells shown are representative of more than 50 scanned cells. 
RhoG-dependent Activation of Rac1 and Cdc42Hs

cells, expression of RhoGN17 (panel c) did not prevent the formation of filopodia (panel d). This demonstrates that RhoG is not involved in either PDGFdependent Rac1 activation or bradykinin-dependent Cdc42Hs activation.

\section{Microtubule Depolymerization Inhibits RhoG but not Rac1 and Cdc42Hs Activities}

As observed in Figure 1A, RhoG protein is distributed within the cytoplasm according to a pattern reminiscent of endoplasmic reticulum membranes, suggesting that RhoG activity might depend on the microtubule network. We thus examined the effect of the microtubule-depolymerizing drug nocodazole on GFPRhoGV12-expressing REF-52 cells (Figure 9A). Cells treated for $30 \mathrm{~min}$ with nocodazole showed a nearly complete disappearance of the microtubule network (compare panels $f$ and $b$ ). During the same time, we observed a reversal of the altered cell morphology (compare panels $\mathrm{e}-\mathrm{h}$ with panels $\mathrm{a}-\mathrm{d}$ ) as well as a relocalization of the RhoG protein, with a concentration in the perinuclear region and a loss at the plasma membrane (arrows in a). The distribution of the RhoGV12 protein in nocodazole-treated cells was similar to that of RhoGN17 (see Figure 1, panels e and f). In addition, the level of stress fibers in all examined GFP-RhoGV12-expressing cells was restored to that observed in untransfected cells (panel h). This inhibitory effect was restricted to RhoG, since cytoskeletal reorganization was not impaired in Rac1V12- and Cdc42HsV12-expressing cells similarly treated. Upon removal of nocodazole and recovery of $60 \mathrm{~min}$ (panels i-1), a microtubule network identical to that detected in control cells (panel j) was observed. Similarly, RhoGV12-transfected cells resumed the same altered morphology as in panels $\mathrm{a}-\mathrm{d}$, and local accumulation of GFP-RhoGV12 was again detected at the plasma membrane (panels i and k). F-actin staining showed the presence of lamellipodia as well as a loss of stress fibers (panel 1). To ascertain that endogenous Rac1 and $\mathrm{Cdc} 42 \mathrm{Hs}$ could still be activated in nocodazole-treated cells, Swiss 3T3 cells were treated for $60 \mathrm{~min}$ with nocodazole (Figure 9B, panel a) before stimulation with either PDGF (panel b) or bradykinin (panel c). PDGF elicited the formation of lamellipodia whereas bradykinin stimulation elicited the production of filopodial extensions, thereby indicating that endogenous Rac1 and Cdc42Hs proteins could be activated with functional consequences even in the absence of a microtubule network. These data therefore demonstrate that the RhoGV12 protein can translocate to the plasma membrane and activate Rac1- and Cdc42Hsdependent cytoskeletal reorganization only in the presence of a microtubule network.

\section{DISCUSSION}

Numerous studies have implicated Rho family members in the control of actin-containing structures (extensively reviewed by Hall, 1998). In many cell types, activation of RhoA leads to stress fibers and focal adhesion formation, activation of Rac1 induces lamellipodia, and adhesion complexes, whereas activation of Cdc42Hs promotes the formation of filopodia and adhesion complexes distinct from Rac1. These activities appear tightly coordinated, as exemplified by the regulatory cascade identified in Swiss 3T3 cells, establishing that activation of Cdc42Hs leads to the subsequent activation of Rac1, which in turn activates RhoA (Nobes et al., 1995). Although most studies have investigated the cellular function of Cdc42Hs, Rac1, and RhoA, the Rho family contains several additional members whose roles in the control of cell morphology have not yet been determined. We previously identified RhoG as a member distantly related to Rac1 and Cdc42Hs (Vincent et al., 1992), which is encoded by a growth-regulated gene (Le Gallic et al., 1997). RhoG protein cooperates in a Rac1-dependent manner with Cdc42Hs in focus formation of NIH3T3 cells, suggesting that RhoG might act upstream of Rac1 (Roux et al., 1997). In this study, we have examined the effects of RhoG expression on the morphology of rat REF-52 and mouse Swiss 3T3 fibroblastic cells as well as the cross-talk among RhoG, Rac1, and Cdc42Hs.

We found that in both cell systems, expression of activated RhoG protein elicited the formation of extensive membrane ruffles and lamellipodia, hallmarks of Rac1 activity, and to a lower extent, filopodial structures, as reported for $\mathrm{Cdc} 42 \mathrm{Hs}$. In addition, expression of activated RhoG, Rac1, and Cdc42Hs induced a decrease in actin stress fibers in $60-80 \%$ of the cells, whereas $\mathrm{Cdc} 42 \mathrm{Hs}$ and RhoG expression also triggered the apparition of numerous microvilli onto the dorsal cell membrane. This is the first report that such apical structures, detected by SEM but not by classical Factin immunofluorescence microscopy, are induced by Cdc42Hs. However, formation of similar microvilli have been recently described in RhoA-expressing cells (Shaw et al., 1998).

To determine the molecular mechanisms underlying the overall morphological effects of RhoG, we used two complementary approaches, based on the use of constitutively active and dominant negative Rho proteins mutants. The activated protein contains a substitution at position 12, resulting in a change from glycine to valine. This mutation is oncogenic in ras genes, and has been shown to decrease the intrinsic GTPase activity of the protein and to make it unresponsive to GTPase-activating proteins (Diekmann et al., 1991). The resulting protein is therefore preferentially bound to GTP and permanently activates its downstream effectors. A dominant negative protein harboring a 
A
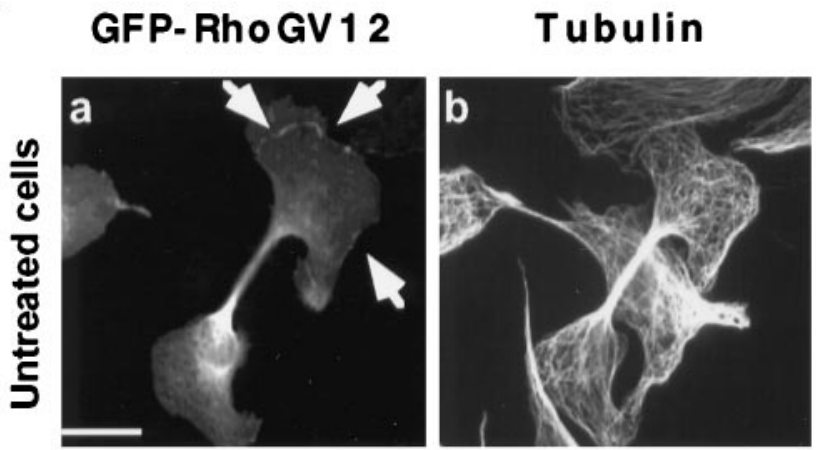

GFP-RhoGV 12
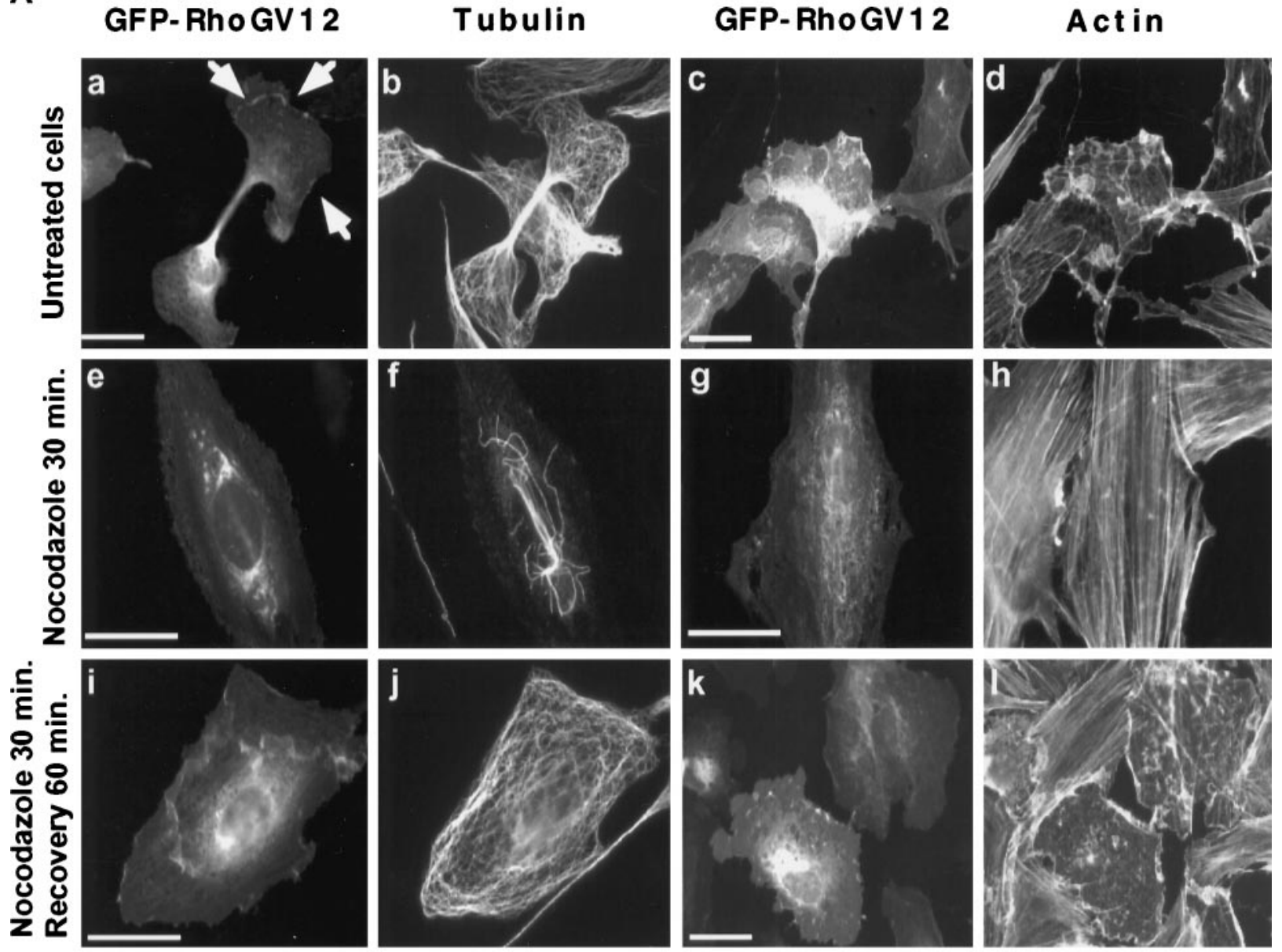

B

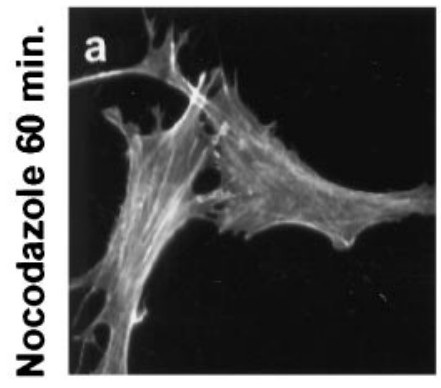

Unstimulated cells

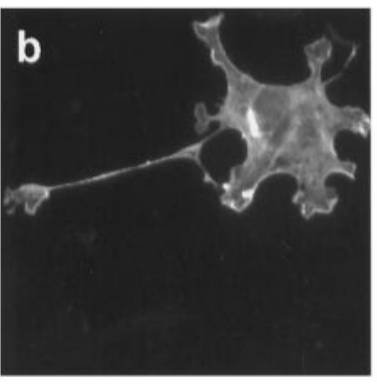

PDGF $10 \mathrm{~min}$.

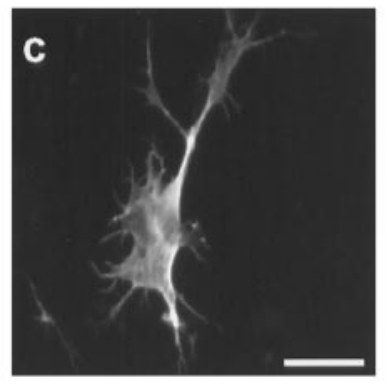

Bradykinin $10 \mathrm{~min}$.

Figure 9. Effect of microtubule depolymerization on GFP-RhoG protein localization and activity. (A) REF-52 cells were transfected with constructs expressing GFP-RhoGV12 protein. Eighteen hours later, transfected cells (panels a-d) were treated for 30 min with $2 \mu \mathrm{M}$ nocodazole (panels e-f). Cells were then rinsed and incubated for $60 \mathrm{~min}$ in media without nocodazole (panels $\mathrm{i}-1)$. Cells were fixed and monitored for GFP activity (panels a, c, e, g, i, and k), microtubule distribution (panels b, f, and j), and F-actin distribution (panels d, h, and 1). Bar, $10 \mu \mathrm{m}$. (B) Swiss $3 \mathrm{~T} 3$ cells were treated for $60 \mathrm{~min}$ with $2 \mu \mathrm{M}$ nocodazole (panel a) and then stimulated for $10 \mathrm{~min}$ with $3 \mathrm{ng} \cdot \mathrm{ml}^{-1}$ PDGF (panel b) or $100 \mathrm{ng} \cdot \mathrm{ml}^{-1}$ bradykinin (panel c). Cells were fixed and observed for F-actin distribution. For each panel, cells shown are representative of more than 100 observed cells. 
substitution at position 17 from threonine to asparagine has decreased affinity for GTP (Feig and Cooper, 1988; Ridley et al., 1992). In contrast to the V12 protein, the N17 protein is unable to bind any effector protein, and, in addition, competitively inhibits the interaction of homologous endogenous GTPbinding proteins with their respective exchange factors (GEFs), thereby preventing the normal activation of downstream targets.

In the first approach, we examined the possibility that RhoGV12 might share downstream effectors with $\mathrm{Rac1}$ and Cdc42Hs. However, interaction assays in the yeast two-hybrid system showed that RhoGV12 did not directly interact with either Rac1 or Cdc42Hs targets, including p65 ${ }^{\mathrm{PAK}}$ (Manser et al., 1994; Martin et al., 1995; Lim et al., 1996), WASP, (Aspenstrom et al., 1996; Kolluri et al., 1996; Symons et al., 1996), POR-1 (Van Aelst et al., 1996), and p160 ${ }^{\text {ROCK }}$ (Fujisawa et al., 1996; Ishizaki et al., 1996; Leung et al., 1996). Rac1 and Cdc42Hs targets have been shown to fall into two main classes, according to their binding domain, p65 ${ }^{\text {PAK }}$ and WASP harboring a CRIB domain [Cdc42/ Rac interactive binding (Burbelo et al., 1995)], and p160 ROCK harboring a REM2 domain [Rho effector motif 2 (Leung et al., 1996)]. In addition, other targets have been characterized that contain neither CRIB nor REM2 domains and interact with the activated GTPases through specific hydrophobic coiled-coil structures. This type of domain is present in POR-1, a Rac1 target involved in membrane ruffling (Van Aelst et al., 1996). Our data in the yeast two-hybrid system suggest that as RhoGV12 does not bind to CRIB, REM2, or POR-1 coiled-coil structures in vitro, it is not likely to be active on most known Rac1 and Cdc42Hs targets.

In the second approach, we directly addressed the requirement of endogenous Rac1 and Cdc42Hs activities by coexpressing T17N dominant negative mutants. In RhoGV12-expressing cells, Rac1N17 inhibited the production of lamellipodia but not filopodia, whereas Cdc42HsN17 prevented the formation of filopodia but not lamellipodia. Conversely, inhibition of endogenous RhoG activity upon expression of RhoGN17 did not modify the ability of Rac1V12 to produce lamellipodia nor Cdc42HsV12 to produce filopodia. This demonstrates that RhoGV12 requires endogenous Rac1 and Cdc42Hs activities to promote the formation of lamellipodia and filopodia, respectively. In addition, RhoG can independently activate Rac1 and Cdc42Hs, since its expression produced Rac1-dependent structures even in the absence of $\mathrm{Cdc} 42 \mathrm{Hs}$ activity. However, these data do not exclude the possibility that Cdc42Hs can activate Rac1, as previously demonstrated in Swiss 3T3 cells (Nobes and Hall, 1995).

Both our approaches therefore support the conclusion that RhoGV12 independently activates Rac1 and
$\mathrm{Cdc} 42 \mathrm{Hs}$ endogenous activities. The activation takes place even in serum-starved cells, which suggests that RhoG triggers a pathway that specifies the GTP-loading of Rac1 and Cdc42Hs. This could be achieved through the activation of GEFs. Similar examples of GTPase/GEF/GTPase cascades have previously been reported for Ost, a Rac1 effector which promotes nucleotide exchange on Cdc42Hs and RhoA (Horii et al., 1994), and for Rlf, a Ras and Rap1A effector acting as a GEF on RalA (Wolthuis et al., 1996). In yeast, the Ras-like GTPase Bud1 has been shown to activate Cdc24, a GEF that in turn activates Cdc42 (Park et al., 1997). Alternatively, the effects of RhoG might be mediated by molecules other than classical GEFs, such as the lipid phosphatidyl-inositol 4,5-bisphosphate, which stimulates Cdc42Hs, RhoA, Rac1, and Arf activities (Zheng et al., 1996). In all instances, RhoG activates Rac1 and Cdc42Hs through a pathway independent from PDGF and bradykinin signaling pathways, since in RhoGN17-expressing cells, these agents still elicit the formation of lamellipodia and filopodia, respectively.

Several arguments support the possibility that the specific pathway controlled by RhoG might involve the microtubule network. Endogenous RhoG protein is distributed according to a microtubule-like pattern and only the GTP-bound form can translocate to the plasma membrane. Indeed, distribution of the dominant negative RhoGN17 remained restricted to the perinuclear region and impaired the localization of the endogenous protein to the plasma membrane. Interestingly, disruption of the microtubule network mimicked the inhibitory effect of RhoGN17, preventing the translocation of endogenous RhoG as well as RhoGV12 to the plasma membrane and inhibiting its morphogenic effects. In contrast, microtubule depolymerization did not affect Rac1V12 and Cdc42HsV12 morphogenic activities nor PDGF- and bradykinindependent membrane ruffling and filopodial extensions. Taken together, these data suggest that the translocation of the GTP-bound form of RhoG to the plasma membrane is required to trigger the activation of Rac1 and Cdc42Hs and this translocation is inhibited by disruption of the microtubule network. Two opposite interpretations can be proposed to connect RhoG activity with the microtubule network. First, inhibition of RhoG activity might be an indirect consequence of microtubule depolymerization. Indeed, microtubule breakdown has been reported to elicit pleitropic effects in serum-starved cells, including the rapid assembly of RhoA-dependent focal adhesions and microfilament bundles (Kajstura and BereiterHahn, 1993; Bershadsky et al., 1996; Lloyd et al., 1997; Zhang et al., 1997). Activation of RhoA following microtubule depolymerization might therefore trigger cell adhesion and contraction which would in turn inhibit RhoG activity. Alternatively, RhoG might use a 
microtubule-dependent transport to translocate to the plasma membrane and produce its morphogenic effects. Inhibition of RhoG activity in nocodazoletreated cells would therefore be a direct consequence of microtubule depolymerization. This latter model better fits our observations that expression of activated RhoG, Rac1, and Cdc42Hs leads to the disassembly of actin bundles in $60-70 \%$ of untreated cells, in agreement with previous studies on $\mathrm{Cdc} 42 \mathrm{Hs}$ (Kozma et al., 1995; Chrzanowska-Wodnicka and Burridge, 1996; Lim et al., 1996). Accordingly, the RhoA-dependent stress fiber formation observed in nocodazole-treated cells would simply reflect a reversion of the phenotype following inhibition of RhoGV12 activity.

The functional analysis of RhoG targets, as well as the identification of upstream regulating factors will help to elucidate the precise mechanisms by which microtubule depolymerization affects RhoG activity, as well as the nature of the RhoG-controlled pathway that results in Rac1 and Cdc42Hs activation.

\section{ACKNOWLEDGMENTS}

We thank P. Chavrier for Rac1 and Cdc42Hs cDNA, J. Camonis for yeast two-hybrid Rac1 and Cdc42Hs vectors, and A. Hall for PAK, ROCK, and WASP constructs. We also thank A. Vie for continuous gift of Swiss 3T3 cells, M. Martin for production and purification of RhoG protein, and B. Hipskind, N. Taylor, and M. Sitbon for critical reading of the manuscript. Confocal microscopy and electron scanning microscopy were performed at the Centre Regional d'Imagerie Cellulaire de Montpellier. This work was supported by institutional grants from the Centre National de la Recherche Scientifique, Institut National de la Santé et de la Recherche Médicale, and University of Montpellier II, and contracts from the Association Francaise contre le Cancer (ARC), Ligue Nationale Contre le Cancer, and CNRS (Cell Biology Project 96033).

\section{REFERENCES}

Adams, A.E., Johnson, D.I., Longnecker, R.M., Sloat, B.F., and Pringle, J.R. (1990). CDC42 and CDC43, two additional genes involved in budding and the establishment of cell polarity in the yeast Saccharomyces cerevisiae. J. Cell Biol. 111, 131-142.

Aepfelbacher, M., Essler, M., Huber, E., Czech, A., and Weber, P.C. (1996). Rho is a negative regulator of human monocyte spreading. J. Immunol. 157, 5070-5075.

Aepfelbacher, M., Vauti, F., Weber, P.C., and Glomset, J.A. (1994). Spreading of differentiating human monocytes is associated with a major increase in membrane-bound CDC42. Proc. Natl. Acad. Sci. USA 91, 4263-4267.

Aspenstrom, P., Lindberg, U., and Hall, A. (1996). Two GTPases, Cdc42 and Rac, bind directly to a protein implicated in the immunodeficiency disorder Wiskott-Aldrich syndrome. Curr. Biol. 6, 7075.

Bershadsky, A., Chausovsky, A., Becker, E., Lyubimova, A., and Geiger, B. (1996). Involvement of microtubules in the control of adhesion-dependent signal transduction. Curr. Biol. 6, 1279-1289.

Braga, V.M., Machesky, L.M., Hall, A., and Hotchin, N.A. (1997). The small GTPases Rho and Rac are required for the establishment of cadherin-dependent cell-cell contacts. J. Cell Biol. 137, 1421-1431.
Brenner, B., Koppenhoefer, U., Weinstock, C., Linderkamp, O., Lang, F., and Gulbins, E. (1997). Fas- or ceramide-induced apoptosis is mediated by a Rac1-regulated activation of Jun N-terminal Kinase/p38 Kinases and GADD153. J. Biol. Chem. 272, 22173-22181.

Brunk, U., Collins, V.P., and Arro, E. (1981). The fixation, dehydration, drying and coating of cultured cells for SEM. J. Microsc. 123, 121-131.

Burbelo, P.D., Drechsel, D., and Hall, A. (1995). A conserved binding motif defines numerous candidate target proteins for both Cdc42 and Rac GTPases. J. Biol. Chem. 270, 29071-29074.

Chardin, P., Camonis, J.H., Gale, N.W., van Aelst, L., Schlessinger, J., Wigler, M.H., and Bar-Sagi, D. (1993). Human Sos1: a guanine nucleotide exchange factor for Ras that binds to GRB2. Science 260, 1338-1343.

Chrzanowska-Wodnicka, M., and Burridge, K. (1996). Rho-stimulated contractility drives the formation of stress fibers and focal adhesions. J. Cell Biol. 133, 1403-1415.

Coso, O.A., Chiariello, M., Yu, J.C., Teramoto, H., Crespo, P., Xu, N.G., Miki, T., and Gutkind, J.S. (1995). The small GTP-binding proteins Rac1 and Cdc42 regulate the activity of the JNK/SAPK signaling pathway. Cell 81, 1137-1146.

Diekmann, D., Brill, S., Garett, M.D., Totty, N., Hsuan, J., Monfries, C., Hall, C., Lim, L., and Hall, A. (1991). Bcr encodes a GTPaseactivating protein for p21rac. Nature 351, 400-403.

Dutartre, H., Davoust, J., Gorvel, J.P., and Chavrier, P. (1996). Cytokinesis arrest and redistribution of actin-cytoskeleton regulatory components in cells expressing the Rho GTPase CDC42Hs. J. Cell Sci. 109, 367-377.

Esteve, P., del Peso, L., and Lacal, J.C. (1995). Induction of apoptosis by rho in NIH $3 \mathrm{~T} 3$ cells requires two complementary signals. Ceramides function as a progression factor for apoptosis. Oncogene 11, 2657-2665.

Feig, L., and Cooper, G.M. (1988). Inhibition of NIH 3T3 cell proliferation by a mutant ras protein with preferential affinity for GDP. Mol. Cell. Biol. 8, 3235-3243.

Fujisawa, K., Fujita, A., Ishizaki, T., Saito, Y., and Narumiya, S. (1996). Identification of the Rho-binding domain of p160ROCK, a Rho-associated coiled-coil containing protein kinase. J. Biol. Chem. 271, 23022-23028.

Gulbins, E., Coggeshall, K.M., Brenner, B., Schlottmann, K., Linderkamp, O., and Lang, F. (1996). Fas-induced apoptosis is mediated by activation of a Ras and Rac protein-regulated signaling pathway. J. Biol. Chem. 271, 26389-26394.

Habets, G.G., Scholtes, E.H., Zuydgeest, D., van der Kammen, R.A., Stam, J.C., Berns, A., and Collard, J.G. (1994). Identification of an invasion-inducing gene, Tiam-1, that encodes a protein with homology to GDP-GTP exchangers for Rho-like proteins. Cell 77, 537-549.

Hall, A. (1998). Rho GTPases and the actin cytoskeleton. Science 279, 509-514.

Hill, C.S., Wynne, J., and Treisman, R. (1995). The Rho family GTPases RhoA, Rac1, and CDC42Hs regulate transcriptional activation by SRF. Cell 81, 1159-1170.

Hirai, A., et al. (1997). Geranylgeranylated rho small GTPase(s) are essential for the degradation of p27Kip1 and facilitate the progression from G1 to $S$ phase in growth-stimulated rat FRTL-5 cells. J. Biol. Chem. 272, 13-16.

Hirata, K., Kikuchi, A., Sasaki, T., Kuroda, S., Kaibuchi, K., Matsuura, Y., Seki, H., Saida, K., and Takai, Y. (1992). Involvement of rho p21 in the GTP-enhanced calcium ion sensitivity of smooth muscle contraction. J. Biol. Chem. 267, 8719-8722.

Horii, Y., Beeler, J.F., Sakaguchi, K., Tachibana, M., and Miki, T. (1994). A novel oncogene, ost, encodes a guanine nucleotide ex- 
change factor that potentially links Rho and Rac signaling pathways. EMBO J. 13, 4776-4786.

Hotta, K., Tanaka, K., Mino, A., Kohno, H., and Takai, Y. (1996). Interaction of the Rho family small G proteins with kinection, an anchoring protein of kinesin motor. Biochem. Biophys. Res. Commun. 225, 69-74.

Ishizaki, T., et al. (1996). The small GTP-binding protein Rho binds to and activates a $160 \mathrm{kDa}$ Ser/Thr protein kinase homologous to myotonic dystrophy kinase. EMBO J. 15, 1885-1893.

Jimenez, B., Arends, M., Esteve, P., Perona, R., Sanchez, R., Ramon, Y., S., C., Wyllie, A., and Lacal, J.C. (1995). Induction of apoptosis in NIH3T3 cells after serum deprivation by overexpression of rho-p21, a GTPase protein of the ras superfamily. Oncogene 10, 811-816.

Joneson, T., McDonough, M., Bar-Sagi, D., and Van Aelst, L. (1996). RAC regulation of actin polymerization and proliferation by a pathway distinct from Jun kinase. Science 274, 1374-1376.

Kajstura, J., and Bereiter-Hahn, J. (1993). Disruption of microtubules induces formation of actin fibrils in density-inhibited 3T3 cells. Cell Biol. Int. 17, 1023-1031.

Khosravi-Far, R., Solski, P.A., Clark, G.J., Kinch, M.S., and Der, C.J. (1995). Activation of Rac1, RhoA, and mitogen-activated protein kinases is required for Ras transformation. Mol. Cell. Biol. 15, 64436453.

Khosravi-Far, R., White, M.A., Westwick, J.K., Solski, P.A., Chrzanowska- Wodnicka, M., Van Aelst, L., Wigler, M.H., and Der, C.J. (1996). Oncogenic Ras activation of Raf/mitogen-activated protein kinase- independent pathways is sufficient to cause tumorigenic transformation. Mol. Cell. Biol. 16, 3923-3933.

Kolluri, R., Tolias, K.F., Carpenter, C.L., Rosen, F.S., and Kirchhausen, T. (1996). Direct interaction of the Wiskott-Aldrich syndrome protein with the GTPase Cdc42. Proc. Natl. Acad. Sci. USA 93, 5615-5618.

Kozma, R., Ahmed, S., Best, A., and Lim, L. (1995). The Ras-related protein $\mathrm{Cdc} 42 \mathrm{Hs}$ and bradykinin promote formation of peripheral actin microspikes and filopodia in Swiss 3T3 fibroblasts. Mol. Cell. Biol. 15, 1942-1952.

Lamaze, C., Chuang, T.-H., Terlecky, L.J., Bokoch, G., and Schmid, S.L. (1996). Regulation of receptor-mediated endocytosis by Rho and Rac. Nature 382, 177-179.

Le Gallic, L., and Fort, P. (1997). Structure of the growth-regulated ARH-G human gene encoding the small GTPase RhoG. Genomics $42,157-160$

Leeuwen, F.N., Kain, H., Kammen, R.A., Michiels, F., Kranenburg, O.W., and Collard, J.G. (1997). The guanine nucleotide exchange factor tiam1 affects neuronal morphology; opposing roles for the small GTPases rac and Rho. J. Cell Biol. 139, 797-807.

Leung, T., Chen, X.Q., Manser, E., and Lim, L. (1996). The p160 RhoA-binding kinase ROK alpha is a member of a kinase family and is involved in the reorganization of the cytoskeleton. Mol. Cell. Biol. $16,5313-5327$.

Leung, T., Manser, E., Tan, L., and Lim, L. (1995). A novel serine/ threonine kinase binding the Ras-related RhoA GTPase which translocates the kinase to peripheral membranes. J. Biol. Chem. 270, 29051-29054.

Lim, L., Manser, E., Leung, T., and Hall, C. (1996). Regulation of phosphorylation pathways by p21 GTPases. The p21 Ras- related Rho subfamily and its role in phosphorylation signalling pathways. Eur. J. Biochem. 242, 171-185.

Lloyd, C.W., Smith, C.G., Woods, A., and Rees, D.A. (1997). Mechanisms of cellular adhesion. II. The interplay between adhesion, the cytoskeleton and morphology of substrate attached cells. Exp. Cell Res. 110, 427-437.
Madaule, P., Furuyashiki, T., Reid, T., Ishizaki, T., Watanabe, G., Morii, N., and Narumiya, S. (1995). A novel partner for the GTPbound forms of rho and rac. FEBS Lett. 377, 243-248.

Manser, E., Leung, T., Salihuddin, H., Zhao, Z.S., and Lim, L. (1994). A brain serine threonine protein kinase activated by $\mathrm{Cdc} 42$ and Rac1. Nature 367, 40-46.

Martin, G.A., Bollag, G., McCormick, F., and Abo, A. (1995). A novel serine kinase activated by rac1/CDC42Hs-dependent autophosphorylation is related to PAK65 and STE20. EMBO J. 14, 1970-1978.

Michiels, F., Habets, G.G., Stam, J.C., van der Kammen, R.A., and Collard, J.G. (1995). A role for Rac in Tiam1-induced membrane ruffling and invasion. Nature 375, 338-340.

Minden, A., Lin, A., Claret, F.X., Abo, A., and Karin, M. (1995). Selective activation of the JNK signaling cascade and c-Jun transcriptional activity by the small GTPases Rac and Cdc42Hs. Cell 81, 1147-1157.

Moorman, J.P., Bobak, D.A., and Hahn, C.S. (1996). Inactivation of the small GTP binding protein Rho induces multinucleate cell formation and apoptosis in murine T lymphoma EL4. J. Immunol. 156, $4146-4153$

Murphy, C., Saffrich, R., Grummt, M., Gournier, H., Rybin, V., Rubino, M., Auvinen, P., Lutcke, A., Parton, R.G., and Zerial, M. (1996). Endosome dynamics regulated by a Rho protein. Nature 384, 427-432.

Nobes, C.D., and Hall, A. (1995). Rho, rac, and cdc42 GTPases regulate the assembly of multimolecular focal complexes associated with actin stress fibers, lamellipodia, and filopodia. Cell 81, 53-62.

Olson, M.F., Ashworth, A., and Hall, A. (1995). An essential role for Rho, Rac, and Cdc42 GTPases in cell cycle progression through G1. Science $269,1270-1272$.

Park, H.O., Bi, E., Pringle, J.R., and Herskowitz, I. (1997). Two active states of the Ras-related Bud1/Rsr1 protein bind to different effectors to determine yeast cell polarity. Proc. Natl. Acad. Sci. USA 94, $4463-4468$.

Qiu, R.-G., Abo, A., McCormick, F., and Symons, M. (1997). Cdc42 regulates anchorage-independent growth and is necessary for Ras transformation. Mol. Cell. Biol. 17, 3449-3458.

Qiu, R.-G., Chen, J., Kirn, D., McCormick, F., and Symons, M. (1995a). An essential role for Rac in Ras transformation. Nature 374, 457-459.

Qiu, R.-G., Chen, J., McCormick, F., and Symons, M. (1995b). A role for Rho in Ras transformation. Proc. Natl. Acad. Sci. USA 92, 1178111785 .

Ridley, A.J., and Hall, A. (1992). The small GTP-binding protein rho regulates the assembly of focal adhesions and actin stress fibers in response to growth factors. Cell 70, 389-399.

Ridley, A.J., and Hall, A. (1994). Signal transduction pathways regulating Rho-mediated stress fibre formation: requirement for a tyrosine kinase. EMBO J. 13, 2600-2610.

Ridley, A.J., Paterson, H.F., Johnston, C.L., Diekmann, D., and Hall, A. (1992). The small GTP-binding protein rac regulates growth factor-induced membrane ruffling. Cell 70, 401-410.

Roux, P., Gauthier-Rouvière, C., Doucet-Brutin, S., and Fort, P. (1997). The small GTPases Cdc42Hs, Rac1 and RhoG delineate Rafindependent pathways that cooperate to transform NIH3T3 cells. Curr. Biol. 7, 629-637. 
Shaw, R.J., Henry, M., Solomon, F., and Jacks, T. (1998). RhoAdependent phosphorylation and relocalization of ERM proteins into apical membrane/actin protrusions in fibroblasts. Mol. Cell Biol. 9, 403-419.

Symons, M., Derry, J.M., Karlak, B., Jiang, S., Lemahieu, V., Mccormick, F., Francke, U., and Abo, A. (1996). Wiskott-Aldrich syndrome protein, a novel effector for the GTPase CDC42Hs, is implicated in actin polymerization. Cell 84, 723-734.

Tapon, N., and Hall, A. (1997). Rho, Rac and Cdc42 GTPases regulate the organization of the actin cytoskeleton. Curr. Opin. Cell Biol. 9, 86-92.

Teramoto, H., Coso, O.A., Miyata, H., Igishi, T., Miki, T., and Gutkind, J.S. (1996). Signaling from the small GTP-binding proteins Rac1 and Cdc42 to the c-Jun N-terminal kinase/stress-activated protein kinase pathway. A role for mixed lineage kinase 3/proteintyrosine kinase 1, a novel member of the mixed lineage kinase family. J. Biol. Chem. 271, 27225-27228.

Van Aelst, L., Joneson, T., and Bar-Sagi, D. (1996). Identification of a novel Rac1 interacting protein involved in membrane ruffling. EMBO J. 15, 3778-3786.
Vincent, S., Jeanteur, P., and Fort, P. (1992). Growth-regulated expression of rhoG, a new member of the ras homolog gene family. Mol. Cell. Biol. 12, 3138-3148.

Vincent, S., and Settleman, J. (1997). The PRK2 kinase is a potential effector target of both Rho and Rac GTPases and regulates actin cytoskeletal organization. Mol. Cell. Biol. 17, 2247-2256.

Wolthuis, R.M., Bauer, B., van't Veer, L.J., de Vries-Smits, A.M., Cool, R.H., Spaargaren, M., Wittinghofer, A., Burgering, B.M., and Bos, J.L. (1996). RalGDS-like factor (Rlf) is a novel Ras and Rap $1 \mathrm{~A}$-associating protein. Oncogene 13, 353-362.

Zhang, Q., Magnusson, M.K., and Mosher, D.F. (1997). Lysophosphatidic acid and microtubule-destabilizing agents stimulate fibronectin matrix assembly through Rho-dependent actin stress fiber formation and cell contraction. Mol. Biol. Cell 8, 1415-1425.

Zheng, Y., Glaven, J.A., Wu, W.J., and Cerione, R.A. (1996). Phosphatidylinositol 4,5-bisphosphate provides an alternative to guanine nucleotide exchange factors by stimulating the dissociation of GDP from Cdc42Hs. J. Biol. Chem. 271, 23815-23819. 